This document is confidential and is proprietary to the American Chemical Society and its authors. Do not copy or disclose without written permission. If you have received this item in error, notify the sender and delete all copies.

\title{
Probing the Differential Dynamics of Monomeric and Dimeric Insulin from Amide-I IR Spectroscopy
}

\begin{tabular}{|c|c|}
\hline Journal: & The Journal of Physical Chemistry \\
\hline Manuscript ID & jp-2019-046288.R1 \\
\hline Manuscript Type: & Article \\
\hline $\begin{array}{r}\text { Date Submitted by the } \\
\text { Author: }\end{array}$ & 22-Jun-2019 \\
\hline Complete List of Authors: & $\begin{array}{l}\text { Desmond, Jasmine; Universitat Basel Departement Chemie, } \\
\text { Koner, Debasish; University of Basel, Chemistry } \\
\text { Meuwly, Markus; Universitat Basel, Chemistry }\end{array}$ \\
\hline
\end{tabular}

\section{SCHOLARONE" Manuscripts}


Probing the Differential Dynamics of

\title{
Monomeric and Dimeric Insulin from Amide-I IR Spectroscopy
}

\author{
Jasmine L. Desmond, Debasish Koner, and Markus Meuwly* \\ Department of Chemistry, University of Basel, Klingelbergstrasse 80, 4056 Basel, \\ Switzerland \\ E-mail: m.meuwly@unibas.ch
}

\begin{abstract}
The monomer $\leftrightarrow$ dimer equilibrium for insulin is one of the essential steps in forming the receptor binding-competent monomeric form of the hormone. Despite this importance, the thermodynamic stability - in particular for modified insulins - is quite poorly understood in part due to experimental difficulties. This work explores 1- and 2-dimensional infrared spectroscopy in the range of the amide-I band for the hydrated monomeric and dimeric wild type hormone. It is found that for the monomer the frequency fluctuation correlation function $(\mathrm{FFCF})$ and the 1d-infrared spectra are position sensitive. The spectra for the -CO probes at the dimerization interface (residues Phe24, Phe25, Tyr26) split and indicate an asymmetry despite the overall (formal) point symmetry of the dimer structure. Also, the decay times of the FFCF for the same-CO probe in the monomer and the dimer can differ by up to one order of magnitude, for example for residue PheB24 which is solvent exposed for the monomer but at the interface for the dimer. The spectroscopic shifts correlate approximately with the average number of hydration waters and the magnitude of the FFCF at time zero. However,


this correlation is only qualitative due to the heterogeneous and highly dynamical environment. Based on density functional theory calculations the dominant contribution for solvent-exposed -CO is found to arise from the surrounding water $(\sim 75 \%)$ whereas the protein environment contributes considerably less. The results suggest that infrared spectroscopy is a positionally sensitive probe of insulin dimerization, in particular in conjunction with isotopic labeling of the probe.

\section{Introduction}

Insulin is a small, aggregating protein with an essential role in regulating glucose uptake in cells. The WT hormone crystallizes as a hexamer consisting of three dimers with either two or four $\mathrm{Zn}$ atoms bound to it.1 The dimer consists of two monomers (chains A and $\mathrm{B}$ with 21 and 30 amino acids, respectively), connected by two inter-chain (Cys ${ }^{\mathrm{A} 7}-\mathrm{Cys}^{\mathrm{B} 7}$, Cys $\left.{ }^{\mathrm{A} 20}-\mathrm{Cys}^{\mathrm{B} 19}\right)$ and one intra-chain $\left(\mathrm{Cys}^{\mathrm{A} 6}-\mathrm{Cys}^{\mathrm{A} 11}\right)$ disulfide bonds. Under physiological conditions the thermodynamically favoured state is the insulin dimer primarily stabilized by hydrogen bonds between residues B24-B26 of one monomer and B26-B24 of the second monomer. ${ }^{1+5}$ However, the functionally relevant state is the monomer which can bind to the insulin receptor. Therefore, dissociation of the dimer is of great physiological importance.

However, experimental data for dimerization free energies of insulin analogues is scarce due to several challenges. The measured dimerization energy for WT insulin is $-7.2 \mathrm{kcal} / \mathrm{mol}$ in favour of the dimer. ${ }^{6}$ Apart from this, it was found that the Gly ${ }^{\mathrm{B} 24}$ mutant does not dimerize in aqueous solution at $\mathrm{pH} 1.9 .^{7}$ Alanine scanning of the dimerization interface showed that the $\mathrm{Ala}^{\mathrm{B} 24}$ analogue is monomeric and does not readily aggregate. ${ }^{89}$ Furthermore, isothermal titration calorimetry (ITC) measurements of N-methylated insulin dimer analogues at positions B24, B25, and B26 $\frac{10 \mid 11}{2}$ revealed considerably reduced (by a factor of 5) dimerization capabilities compared with human insulin. Also, using computations the dimerization free energies for the WT dimer $\stackrel{412}{ }$ and, more recently, for several mutants at position B24 
were determined. 13

The current view of the insulin structure-function relationship is derived primarily from insulin hexamer and dimer crystal structures, as well as from studies of the structureactivity correlations of chemically modified and/or naturally occurring mutant insulins in solution. $714-21$ Mutagenesis of the dimer-forming surface of insulin can yield analogues with a reduced tendency to aggregate and pronounced differences in the pharmacokinetic properties with potentially promising therapeutic applications. ${ }^{1422}$ Typical experimental methods for quantitative studies of insulin dimerization are ITC ${ }^{3}$ or NMR spectroscopy. ITC requires relatively high protein concentrations, while NMR spectroscopy can be (too) slow for such purposes. Based on transition state theory a free energy difference of $-7.2 \mathrm{kcal} / \mathrm{mol}$ between the WT dimer and two separate monomers corresponds to a sub- $\mu$ s time scale. Several NMR studies of active monomeric insulin mutants show a rearrangement of the C-terminal end of chain B. $\stackrel{720}{ }$ A combined Raman spectroscopy and microscopy study of insulin in different aggregation states (monomer, dimer, hexamer and fibril) shows that dimerization damps fluctuations at an intermolecular $\beta$-sheet. ${ }^{23}$ More recently, vibrational spectroscopy has also been used to characterize the insulin dimer ${ }^{24}$ and isotopically labelled insulins. $\frac{25}{2}$

Earlier experiments on model peptides in solution (water), such as the blocked alanine trimer $(\mathrm{Ala})_{3}$ already found that the two amide-I bands appear at positions $25 \mathrm{~cm}^{-1}$ apart. ${ }^{26}$ Similar observations have been made for acyl-proline- $\mathrm{NH}_{2}$ in chloroform for which the two oscillators absorb at maximum frequencies $40 \mathrm{~cm}^{-1}$ to $50 \mathrm{~cm}^{-1}$ apart depending on the time delay in the 2-dimensional infrared experiments. $\frac{27}{27}$ Therefore, it is expected that by increasing the number of reporters, e.g. in a protein with one amide-I probe per amino acid, the -CO absorption bands will partly overlap and cover an even larger range due to differing chemical environments that are probed during the dynamics. 
Given the fundamental importance of insulin dimerization it is desirable to develop methodologies that are sensitive to and provide direct information on the aggregation state of the hormone in solution. In the present work vibrational spectroscopy is used for that. Infrared spectroscopy (IR) has been shown to be environment specific. For example, the CH-stretch in $\mathrm{HCO}^{+}$is known to shift depending on the number of surrounding argon atoms. ${ }^{28} \mathrm{In}$ $\mathrm{Ar}-\mathrm{HCO}^{+}$the red shift is $-274 \mathrm{~cm}^{-1}$ whereas for each additional Ar atom an incremental blue shift by $\sim 10 \mathrm{~cm}^{-1}$ is observed. For the first complete solvation sphere, containing 12 Argon atoms, the red shift relative to the gas phase reduces to $-155 \mathrm{~cm}^{-1}$. Hence vibrational spectroscopy is a sensitive environmental probe of a spectroscopic reporter.

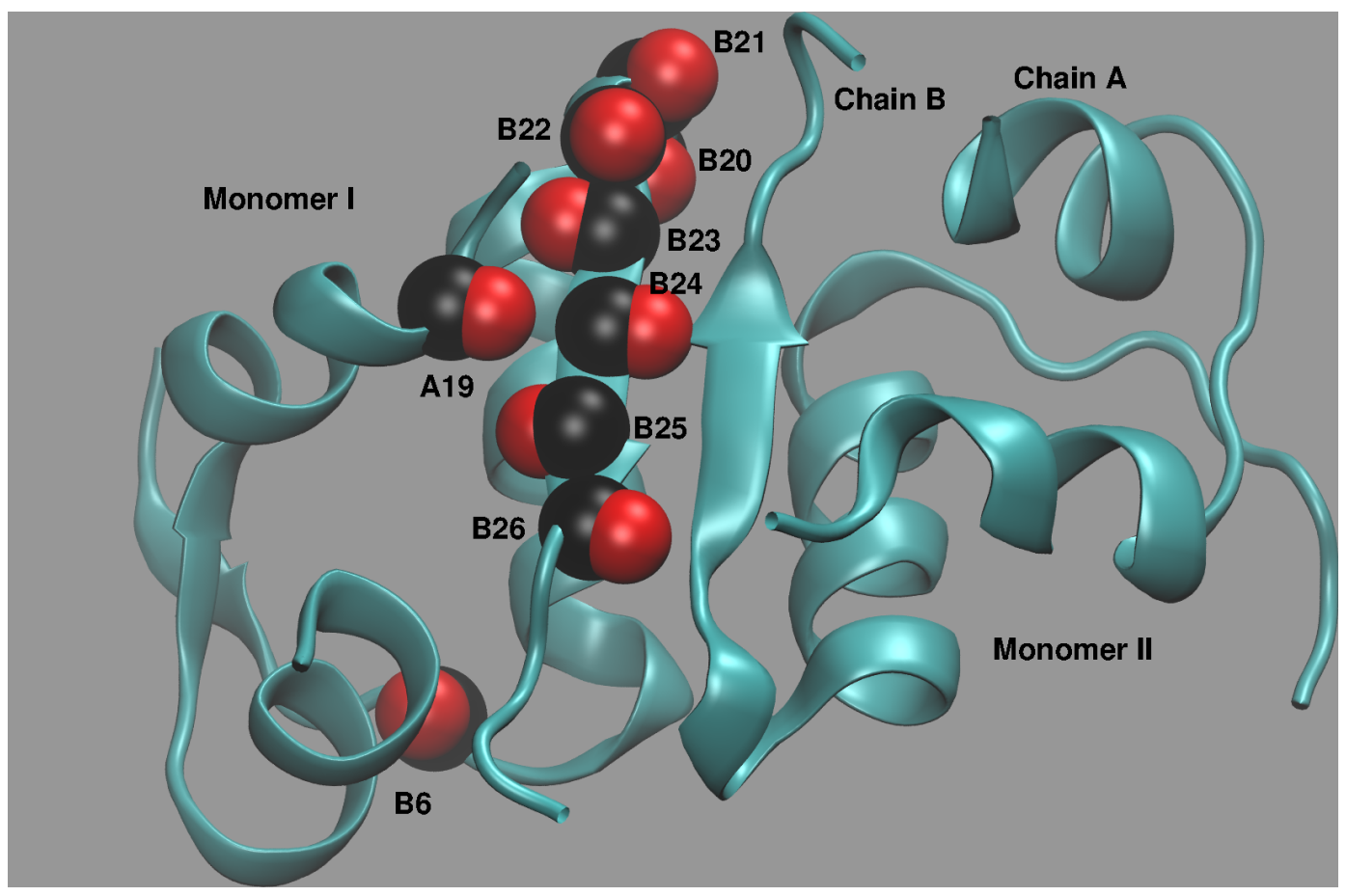

Figure 1: Structure of insulin dimer (PDB Code: 4INS) consisting of monomer I and II, each with chains A and B. Some -CO probes are explicitly represented as van der Waals spheres and labelled.

Because the monomer $\leftrightarrow$ dimer equilibrium is one of the essential steps in forming the receptor binding-competent monomeric form of insulin and the process is difficult to study quantitatively by experiments, MD simulations are an attractive alternative to characterize 
The frequency trajectory of a local reporter can be followed in different ways. One of them uses so-called parametrized "frequency maps", pioneered by Skinner and coworkers, which are precomputed for a given reporter from a large number of ab initio calculations. ${ }^{30131}$ Such maps are very convenient and, once parametrized, computationally advantageous. Much has been learned from them on the multidimensional spectroscopy of water and for peptides and proteins in the range of the amide-I band ${ }^{30 \mid 32}$ To develop such a map, snapshots from MD simulations are used. The frequencies of the reporter are computed using electronic structure methods (e.g. B3LYP $\left./ 6-311++\mathrm{G}^{* *}\right)^{31}$ by stretching and compressing the bond of interest to map out a 1-dimensional potential energy curve from which the 1-0 and 2-1 transitions are calculated by solving the 1-dimensional Schrödinger equation. At the same time, the strength of the electric field at the water hydrogen atom (for water-OH maps) ${ }^{31}$ is determined and the frequency and field strength are treated as a set. The ensuing, empirical correlation is then fit to a parametric function which allows to determine the expected frequency of the parametrized oscillator from the knowledge of the strength of the electric field alone. 
While convenient, such a treatment also introduces approximations. The frequency shift for given field strength has typically a finite width which can be up to $50 \mathrm{~cm}^{-1}$ for $\mathrm{OH} \cdot \stackrel{31}{3} \mathrm{Hence}$, such a map can be regarded as a "mean field approximation" as the fluctuations are usually disregarded. Often, the field is assumed to be linear along the probe, although exceptions exist. ${ }_{33}$ As the electric fields on molecular length scales can be strong, corrections due to the interaction of the charges with the field gradient and higher order terms may be required for an improved relationship between oscillator frequency and field strength. The clusters used in the electronic structure calculations are often quite small (e.g. $4 \AA$ within the probe with implicit inclusion of additional waters up to $8 \AA)^{31}$ out of necessity to keep the electronic structure calculations at a manageable level. In practice, the maps are fitted to reproduce experimentally determined line positions but the final models are transferable to some degree. ${ }^{32}$ A potential matter of concern of such maps is the fact that the nuclear dynamics is followed with an energy function that is typically that of an empirical force field whereas the analysis of the snapshots is carried out at a different level of theory. Thus, there is a conceptual difficulty in that the distribution of conformations from the MD simulations is not necessarily consistent with that one would obtain if sampling would have been carried out at the DFT or even MP2 level of theory which is the level at which the maps are determined.

Alternatively, the sampling of the configurations and computing frequencies for given snapshots can also be done by using the same energy function ("scan"). This has been pursued in the past in the context of multipolar force fields and applied to systems such as hydrated $\mathrm{CN}^{-}, \underline{34}$ the $-\mathrm{CO}$ probe in N-methyl acetamide, $\stackrel{35}{35}$ or $\mathrm{N}_{3}^{-}$in the gas phase and in solution. In this approach the MD simulations are carried out with the same energy function that is also used for the analysis, which is typically a multipolar or a fluctuating charge representation for the electrostatics around the spectroscopic probe and an anharmonic (Morse) or even superior reproducing kernel $\mathrm{PES}\left[\frac{36-38}{38}\right.$ for the bonded terms (stretch and bend). On each snapshot the local frequency is determined from either an instantaneous normal mode 


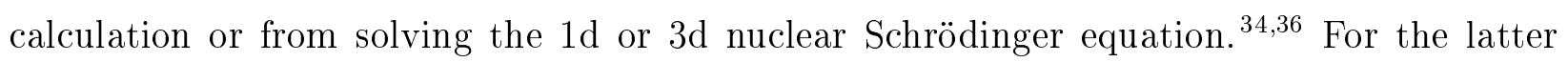
approach, the PES is scanned along all relevant degrees of freedom, similar to the map approach. However, when solving the Schrödinger equation, choosing the reduced mass is not obvious, in particular for covalently bound probes.

For $\mathrm{CN}^{-}$in solution, decay times of the FFCF and the full width at half maximum are in quantitative agreement with experiment using such an approach. ${ }^{34}$ Similarly, for NMA in solution the decay times of the FFCF have been found to agree well with experiment, whereas the full width at half maximum is underestimated $\left(14 \mathrm{~cm}^{-1}\right.$ compared with $\left.29 \mathrm{~cm}^{-1}\right) .[35$ As a comparison, a more recent study of the dynamics of NMAD in $\mathrm{D}_{2} \mathrm{O}$ based on a parametrized DFT map finds a FWHM of $43 \mathrm{~cm}^{-1}$ which overestimates the experimentally determined value. ${ }^{39}$ Conversely, a more elaborate procedure which fits ${ }^{32}$ the map to experimental data in different solvents yields a FWHM of $34 \mathrm{~cm}^{-1}$.

Overall, both approaches ("map" and "scan") make their own approximations and neither of them is strictly "exact". In the following, we use instantaneous normal modes and the explicit solution of the nuclear Schrödinger equation to follow the frequency trajectory of the oscillator in the field of its environment.

This article is organized as follows: Section 2 introduces the methods. In Section 3 first results on the spectroscopy of hydrated N-Methyl-Acetamide (NMA) in solution are reported. This is followed by the discussion of the frequency fluctuation correlation functions and lineshapes of -CO in monomeric and dimeric insulin in solution. Finally, conclusions are drawn in Section 4. 


\section{Methods}

\subsection{Molecular Dynamics Simulations}

All molecular dynamics (MD) simulations were carried out using the CHARMM MD simulation package, $\underline{40}$ with the CHARMM22 force-field. 11 The X-ray crystal structure of the insulin dimer $(\mathrm{PDB} \text { code } 4 \mathrm{INS})^{1}$ was solvated in a cubic box $\left(52.77 \times 52.77 \times 52.77 \AA^{3}\right)$ of TIP3P ${ }^{42}$ water molecules, which leads to a total system size of $\sim 17500$ atoms. For the monomer simulations chains $\mathrm{A}$ and $\mathrm{B}$ were retained and also solvated in a water box $\left(52.77 \times 52.77 \times 52.77 \AA^{3}\right.$, the same box size as the dimer). Hydrogen atoms were included using CHARMM. The systems were neutralised with potassium ions (one for the monomeric system and two for the dimer).

The systems were minimised, heated to the relevant temperature for $50 \mathrm{ps}$ and equilibrated for $250 \mathrm{ps}$ in the NVT ensemble. Production data (up to $4 \mathrm{~ns}$ ) was generated in the NPT ensemble, with coordinates saved every 5 fs for subsequent analysis. All bonds involving hydrogen atoms were constrained using SHAKE. ${ }^{43}$ A Velocity Verlet integrator ${ }^{44}$ and NoseHoover thermostat $\frac{45 \mid 46}{46}$ were employed in the $N V T$ simulations. For the NPT simulations an Andersen and Nose-Hoover constant pressure and temperature algorithm was used $\underline{46-48}$ together with a leapfrog integrator. ${ }^{49}$ Non-bonded interactions were treated with a switching function between $10 \AA$ and $14 \AA .50$ The Particle Mesh Ewald (PME) method was used for the electrostatic interactions. .51

Two simulations of N-methyl acetamide (NMA) - featuring an amide bond with (1) natural ${ }^{12} \mathrm{C}^{16} \mathrm{O}$ isotopes and (2) heavy ${ }^{13} \mathrm{C}^{18} \mathrm{O}$ isotopes - were carried out to validate the computational procedures. In these simulations the entire NMA molecule was treated with multi-

poles. $\sqrt[35 \sqrt{52}-\sqrt{54}]{ }$ For the bonded terms the parameters were those of the CHARMM22 force field except for the -CO stretch for which a Morse potential $V(r)=D_{e}\left(1-\exp \left(-\beta\left(r-r_{e}\right)\right)\right)^{2}$ was 
used. The parameters are $D_{e}=120.47 \mathrm{kcal} / \mathrm{mol}, \beta=2.174 \AA^{-1}$ and $r_{0}=1.294 \AA$ which reproduce the experimental gas phase $\mathrm{C}=\mathrm{O}$ stretch frequency of $1731 \mathrm{~cm}^{-1} . \underline{3555}$

\subsection{Calculation of the $1 \mathrm{D}$ infrared spectra}

The anharmonic frequencies were computed from the time-ordered snapshots extracted from the MD simulation every 5 fs. For this purpose, 1-dimensional potential energy curves were calculated along the -CO bond for each snapshot by freezing all other atoms of the system. The anharmonic transition frequencies $(v=0 \rightarrow 1)$ were then calculated by solving the $1 \mathrm{D}$ time-independent Schrödinger equation using the LEVEL ${ }^{56}$ program to obtain the frequency trajectory. Following this, the frequency fluctuation correlation function, $C(t)=$ $\langle\delta \omega(0) \delta \omega(t)\rangle$, was calculated. Here, $\delta \omega(t)=\omega(t)-\langle\omega(t)\rangle$, where $\langle\omega(t)\rangle$ is the ensemble average. The lineshape function $g(t)$ is determined from the FFCF within the cumulant approximation as,

$$
g(t)=\int_{0}^{t} \int_{0}^{\tau^{\prime}}\left\langle\delta \omega\left(\tau^{\prime \prime}\right) \delta \omega(0)\right\rangle d \tau^{\prime \prime} d \tau^{\prime}
$$

Depending on how $C(t)$ is represented, the integral in Eq. 1 can be evaluated analytically. Two parametrizations were considered for this, depending on the shape of $C(t)$ : either a superposition of exponentially decaying functions 58

$$
C(t)=\sum_{i=1}^{2} a_{i} e^{-t / \tau_{i}}+\Delta_{0}
$$

or one including a recurrence term 59

$$
C(t)=a_{1} \cos (\gamma t) e^{-t / \tau_{1}}+\sum_{i=2}^{3} a_{i} e^{-t / \tau_{i}}+\Delta_{0}
$$

where the $a_{i}$ are amplitudes, the $\tau_{i}$ the decay constants and $\Delta_{0}$ a constant for cases in which $C(t)$ does not decay to zero for the correlation times considered. The decay times $\tau_{i}$ of the 
frequency fluctuation correlation function reflect the characteristic time-scales of the solvent fluctuations to which the solute degrees of freedom are coupled.

Finally, the 1D IR spectra is calculated as

$$
I(\omega)=2 \Re \int_{0}^{\infty} e^{i(\omega-\langle\omega\rangle) t} e^{-g(t)} e^{-\frac{t \alpha}{2 T_{1}}}
$$

where $\langle\omega\rangle$ is the average transition frequency obtained from the distribution, $T_{1}=0.45 \mathrm{ps}$ is the vibrational relaxation time and $\alpha=0.5$ is a phenomenological factor to account for lifetime broadening. $\underline{57}$

The current analysis makes a few assumptions in calculating the FFCF and the 1D IR spectra. Using the cumulant approximation was thoroughly tested within the present framework for $\mathrm{CN}^{-}$in solution for which the lineshapes from analysis with and without the cumulant approximation differ by less than $10 \%$. Since $\mathrm{CN}^{-}$in solution is more strongly affected by coupling between rotation and vibration (approximations in the orientational dynamics), this effect is expected to be smaller in the present case. Furthermore, previous work has found that non-Condon effects for amide-I vibrations are small. 3960

\section{Results and Discussion}

\subsection{NMA (N-Methylacetamide) in Water}

In order to validate the present computational approach, simulations of N-Methylacetamide (NMA) in water for the natural $\left(\mathrm{C}^{12} \mathrm{O}^{16}\right)$ and the heavy $\left({ }^{13} \mathrm{C}^{18} \mathrm{O}\right)$ isotopes in the amide bond were carried out. NMA is a suitable model for the peptide linkages of the protein backbone and therefore a meaningful proxy. Previous experimental measurements of the CO absorption band of NMA in the gas phase found absorptions in the range of $1723 \mathrm{~cm}^{-1}$ 
to $1731 \mathrm{~cm}^{-1}$ (average of $\left.\sim 1725 \mathrm{~cm}^{-1}\right)^{[55[61 \mid 62}$ which shifts to the red $\left(1617 \mathrm{~cm}^{-1}\right.$ to 1625 $\left.\mathrm{cm}^{-1}\right)^{62}$ for NMA in solution. Hence, the experimentally observed red shift between gasand condensed-phase is $\sim 100 \mathrm{~cm}^{-1}$. Previous simulations in which NMA was kept frozen in the simulations and the frequency trajectories were determined from scanning the potential energy for each snapshot along the amide-I normal mode found a red shift of $19 \mathrm{~cm}^{-1} \underline{35}$

In the present work the chromophores are fully flexible and frequencies were determined either from an instantaneous normal mode (INM) analysis (for NMA) or from solving the 1-dimensional Schrödinger equation (for NMA and the -CO probes in insulin monomer and dimer) for the potential energy scanned along the -CO local mode. In NMA, there are two normal modes with large contributions of the -CO stretch; one at $1592 \mathrm{~cm}^{-1}$ (see Figure $2 \mathrm{~B}$ ) and the second one at $1801 \mathrm{~cm}^{-1}$ (Figure S1). Although the second mode is closer in frequency to the experimentally observed band,,$\frac{55}{5}$ the first mode was followed as its -CO local mode character was larger when analyzing the normal modes of NMA in solution. However, as only relative frequency shifts depending on the environmental dynamics are of interest in the present work the results are not expected to depend on this choice when using the instantaneous normal mode analysis.

The power spectrum for NMA in the gas phase is reported in Figure 2A. For the natural isotope (green) the maximum peak frequency is at $1724 \mathrm{~cm}^{-1}$, in good agreement with experiment, which shifts to $1698 \mathrm{~cm}^{-1}$ for simulations in water (black) and corresponds to a solvent-induced red shift of $-26 \mathrm{~cm}^{-1}$. The isotope shift (red) for simulations in solution is $65 \mathrm{~cm}^{-1}$, compared with $60 \mathrm{~cm}^{-1}$ from experiment. $\frac{65}{65}$ The absolute red shift in going from the gas phase into solution for the amide-I vibration between computations $\left(\sim 30 \mathrm{~cm}^{-1}\right)$ and experiment $\left(\sim 100 \mathrm{~cm}^{-1}\right)$ differs by about $70 \mathrm{~cm}^{-1}$. This can be explained by the fact that in the present simulations the -CO stretch potential for simulations in the gas- and condensed-phase is identical. Thus, the computed frequency shift is entirely due to the differ- 

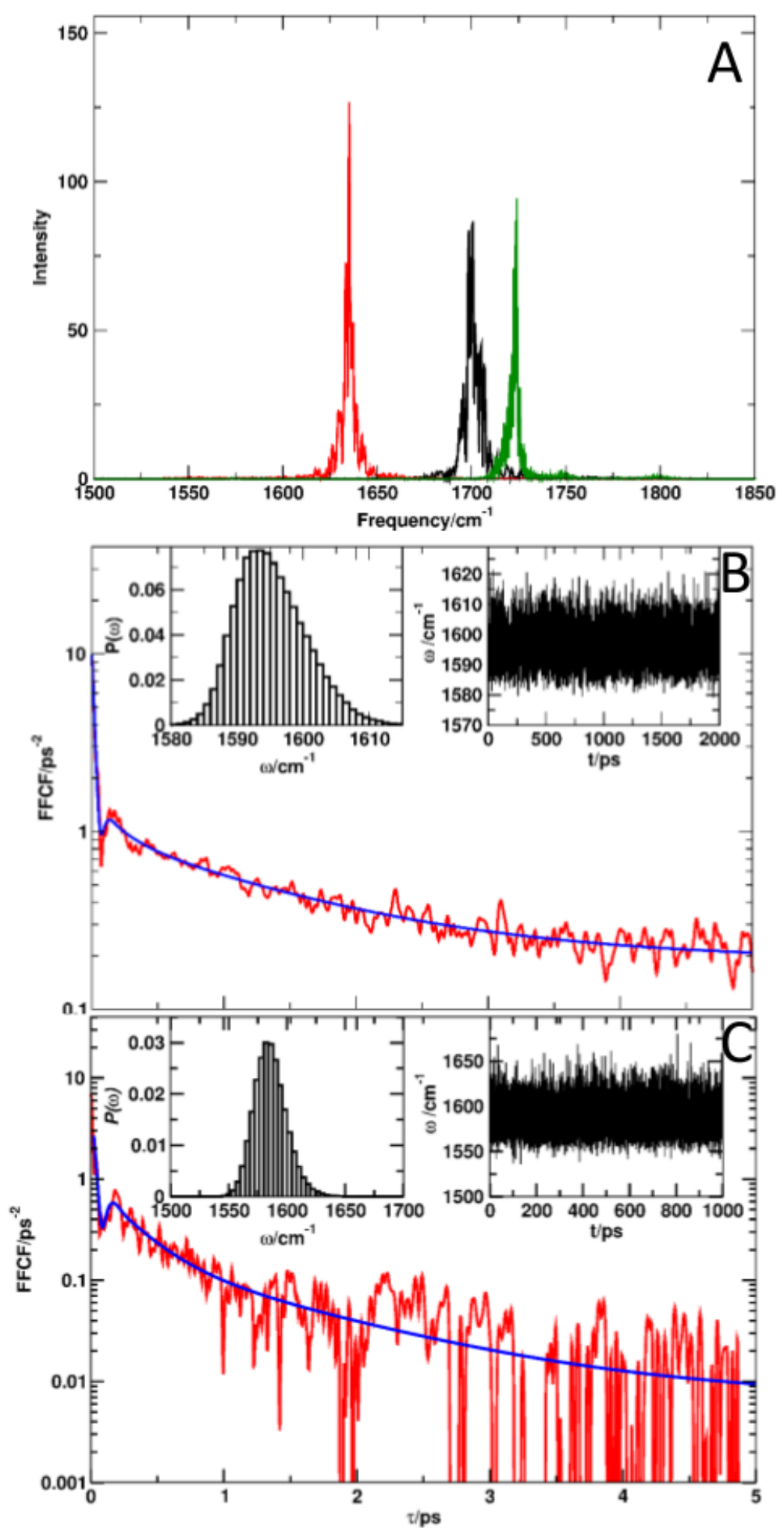

Figure 2: Power spectra and frequency fluctuation correlation functions (FFCFs) for NMA. Panel A: power spectra for ${ }^{12} \mathrm{C}^{16} \mathrm{O}$ (green, in gas phase, $\nu_{\max }=1723 \mathrm{~cm}^{-1}$ ), ${ }^{12} \mathrm{C}^{16} \mathrm{O}$ (black, in solution, $\nu_{\max }=1700 \mathrm{~cm}^{-1}$ ) and ${ }^{13} \mathrm{C}^{18} \mathrm{O}\left(\mathrm{red}, \nu_{\max }=1635 \mathrm{~cm}^{-1}\right.$ ). Panel B: FFCF from INM analysis for ${ }^{12} \mathrm{C}^{16} \mathrm{O}$. The insets report the frequency distribution and frequency trajectory $\omega(t)$. Panel C: FFCF with frequencies calculated using LEVEL for ${ }^{12} \mathrm{C}^{16} \mathrm{O}$ together with the frequency distribution and the frequency trajectory. The parameters used for the fits are given in Table 1. In panels B and $\mathrm{C}$ the red lines are the raw data and the blue lines are the fit. 
ent electrostatic interactions in the two environments. It is, however, expected that the $-\mathrm{CO}$ stretch potentials in the gas- and condensed phase slightly differ which could, in principle, be captured by the parametrization. As an illustration, changing the repulsive parameter $\beta$ in the Morse potential from $2.174 \AA^{-1}$ by $\sim 0.12 \AA^{-1}$ to $2.050 \AA^{-1}$ shifts the maximum of the power spectrum in solution by $-70 \mathrm{~cm}^{-1}$, to the value known from experiment. Likewise, changing the van der Waals radii of the carbon and oxygen atoms by $5 \%$ shifts the absorption frequency by $\pm 15 \mathrm{~cm}^{-1}$. Nevertheless, because in the present work relative frequency shifts in the same environment (water) are of interest this was not pursued further.

Computing frequencies from INM or from scanning the CO-potential in the solvent environment and solving the 1-dimensional Schrödinger equation provides alternative ways to construct the frequency trajectory $\omega(t)$ from which the frequency fluctuation correlation function $(\mathrm{FFCF})$ is determined. Both methods make somewhat different approximations. When using instantaneous normal modes, the harmonic approximation is assumed and the motions are not necessarily local, i.e. a particular normal mode will contain CO stretch motion coupled to other internal degrees of freedom. On the other hand, solving for the stationary states by scanning the CO-potential assumes a local mode and requires one to choose a reduced mass which, however, only affects the absolute frequencies but leaves relative blue- and red-shifts unchanged. To further test this, the frequency distribution was analyzed from solving the 1-dimensional Schrödinger equation for $10^{5}$ snapshots by scanning the potential along the amide-I normal mode. The frequency distribution (see Figure S2) peaks at $1645 \mathrm{~cm}^{-1}$ which is shifted by $20 \mathrm{~cm}^{-1}$ to the blue compared with experiment at $1625 \mathrm{~cm}^{-1}$.

The maximum of the frequency distribution $P(\omega)$ calculated using INM for NMA in water (Figure 2B) is at $1594 \mathrm{~cm}^{-1}$. For the frequency trajectory from solving the 1D Schrödinger equation (Figure $2 \mathrm{C}$ ) the maximum is found at $1586 \mathrm{~cm}^{-1}$. In these calculations the reduced 
Table 1: Fitting parameters from exponential fits of the FFCFs for ${ }^{12} \mathrm{C}^{16} \mathrm{O}$ and ${ }^{13} \mathrm{C}^{18} \mathrm{O}$ in NMA. Amplitudes $a_{i}$ and offset $\Delta_{0}$ are reported in $\mathrm{cm}^{-2}, \tau_{i}$ are in ps and $\gamma$ in $\mathrm{ps}^{-1}$. The short time scale from the experiments in Ref. ${ }^{29}$ (50 to $100 \mathrm{fs}$ ) was estimated. In Ref. ${ }^{35}$ only a sum of three exponentials was used to fit the FFCF.

\begin{tabular}{l|lll|ll|ll|l}
\hline \hline & $a_{1}$ & $\tau_{1}$ & $\gamma$ & $a_{2}$ & $\tau_{2}$ & $a_{3}$ & $\tau_{3}$ & $\Delta_{0}$ \\
& $\mathrm{ps}^{-2}$ & $\mathrm{ps}$ & $\mathrm{ps}^{-1}$ & $\mathrm{ps}^{-2}$ & $\mathrm{ps}$ & $\mathrm{ps}^{-2}$ & $\mathrm{ps}$ & $\mathrm{ps}^{-2}$ \\
\hline $\mathrm{INM}^{[35]}$ & 3.89 & 0.07 & - & 1.04 & 0.42 & 1.01 & 1.07 & - \\
mode 33 (INM) & 8.38 & 0.03 & 32.41 & 0.58 & 0.18 & 0.79 & 1.34 & 0.19 \\
${ }^{12} \mathrm{C}^{16} \mathrm{O}(1 \mathrm{~d}-\mathrm{SE})$ & 3.79 & 0.04 & 29.38 & 0.77 & 0.24 & 0.22 & 1.04 & 0.01 \\
\hline Exp. $\frac{[29}{\text { Exp. }[6]}$ & & $(0.05-0.1)$ & & & & & 1.6 & \\
\hline
\end{tabular}

mass was that of either ${ }^{12} \mathrm{C}^{16} \mathrm{O}$ or ${ }^{13} \mathrm{C}^{18} \mathrm{O}$. For the power spectra (Figure $2 \mathrm{~A}$ ) the isotope shift is $65 \mathrm{~cm}^{-1}$ (compared with $60 \mathrm{~cm}^{-1}$ from experiment) $\frac{65}{6}$ wereas for the spectra from solving the 1D Schrödinger equation (Figures $2 \mathrm{C}$ ) it is $74 \mathrm{~cm}^{-1}$.

The FFCF for the INM and quantum bound state frequencies are reported in Figures $2 \mathrm{~B}$ and C. At very short time $(\tau \sim 0.1 \mathrm{ps})$ a minimum in the FFCF is found which was not present in earlier work ${ }^{35}$, probably due to the fact that the solute was frozen during the simulations. This feature is known from previous simulations ${ }^{67}$ and related to the strength of the interac-

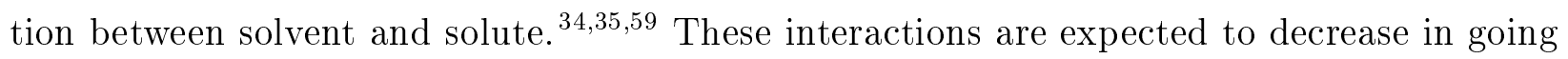
from $\mathrm{CN}^{-}, \mathrm{N}_{3}^{-}, \mathrm{H}_{2} \mathrm{O}$ to the amide-I in N-methyl-acetamide due to the different strengths of the dipole and higher multipolar interactions with the environment. The two FFCFs show invariably multiexponential time decays. The parameters of the fits to Eqs. 2 and 3 are provided in Table 1 and compared with earlier work and experiment. ${ }^{29 \mid 66}$ The quality of the fits is good and the time decays $\tau_{3}$ derived from them are consistent with the experiments which validate the computational model for using it in simulations of insulin monomer and dimer. 


\subsection{FFCF and Infrared Spectra for the Insulin Monomer}

Next, the FFCFs and infrared spectra of -CO probes for insulin monomer in water were determined. For this, $2 \times 10^{5}$ snapshots, separated by 5 fs were analyzed from the MD simulations and the positionally resolved spectra were determined from analytically integrating the corresponding FFCFs, Eqs. 2 and 3. In all cases the FFCF was fitted either to Eq2 2 or 3. depending on whether the minimum in the FFCF at short time $(\tau \leq 0.1 \mathrm{ps})$ was present or not.

Typical FFCFs are reported in Figure $3 \mathrm{~A}$. The FFCFs for -CO probes at different positions along the polypeptide chain (see Figure 1) show that the local environmental dynamics differs. This is evident from changes in the curvature, the static components (as indicated by the value of $C(t=5 \mathrm{ps}))$, and the fact that some of the FFCFs have a minimum at short correlation times whereas others do not. The fact that some of the -CO probes exhibit a weak minimum whereas others do not suggests that the simulations are sensitive to such different probe/solvent interactions and will be discussed further below.

More specifically, the correlation functions for the -CO probes at residues A19, B6, B20, and B24 for the monomer together with the fits to the parametrized representations (Eqs. 2 and 3) are reported in Figure $3 \mathrm{~A}$. The initial amplitude $C(t=0)$ ranges from $4 \mathrm{ps}^{-2}$ for B6 to $\sim 8 \mathrm{ps}^{-2}$ for the other three residues considered. Similarly, the asymptotic value (here taken at $5 \mathrm{ps)} \mathrm{differs} \mathrm{for} \mathrm{B6} \mathrm{compared} \mathrm{with} \mathrm{the} \mathrm{other} \mathrm{three} \mathrm{residues.} \mathrm{For} \mathrm{A19,} \mathrm{B20,} \mathrm{and} \mathrm{B24} \mathrm{the}$ FFCF at longer times decays to a value close to zero whereas that for B6 remains at a finite value of $\sim 1 \mathrm{ps}^{-2}$, see inset of Figure 3 A. Finally, at short times $(t \sim 0.1 \mathrm{ps})$ the FFCFs for B6 (faint) and B20 (pronounced, see lower inset Figure 3A) exhibit a minimum, that for A19 displays a shoulder whereas that for B24 has no discernible feature. All FFCFs clearly contain multiple time scales and the fits to the empirical representations are of good quality. Overall, the FFCFs for -CO at different locations along the polypeptide chain show different, 
clearly distinct features.
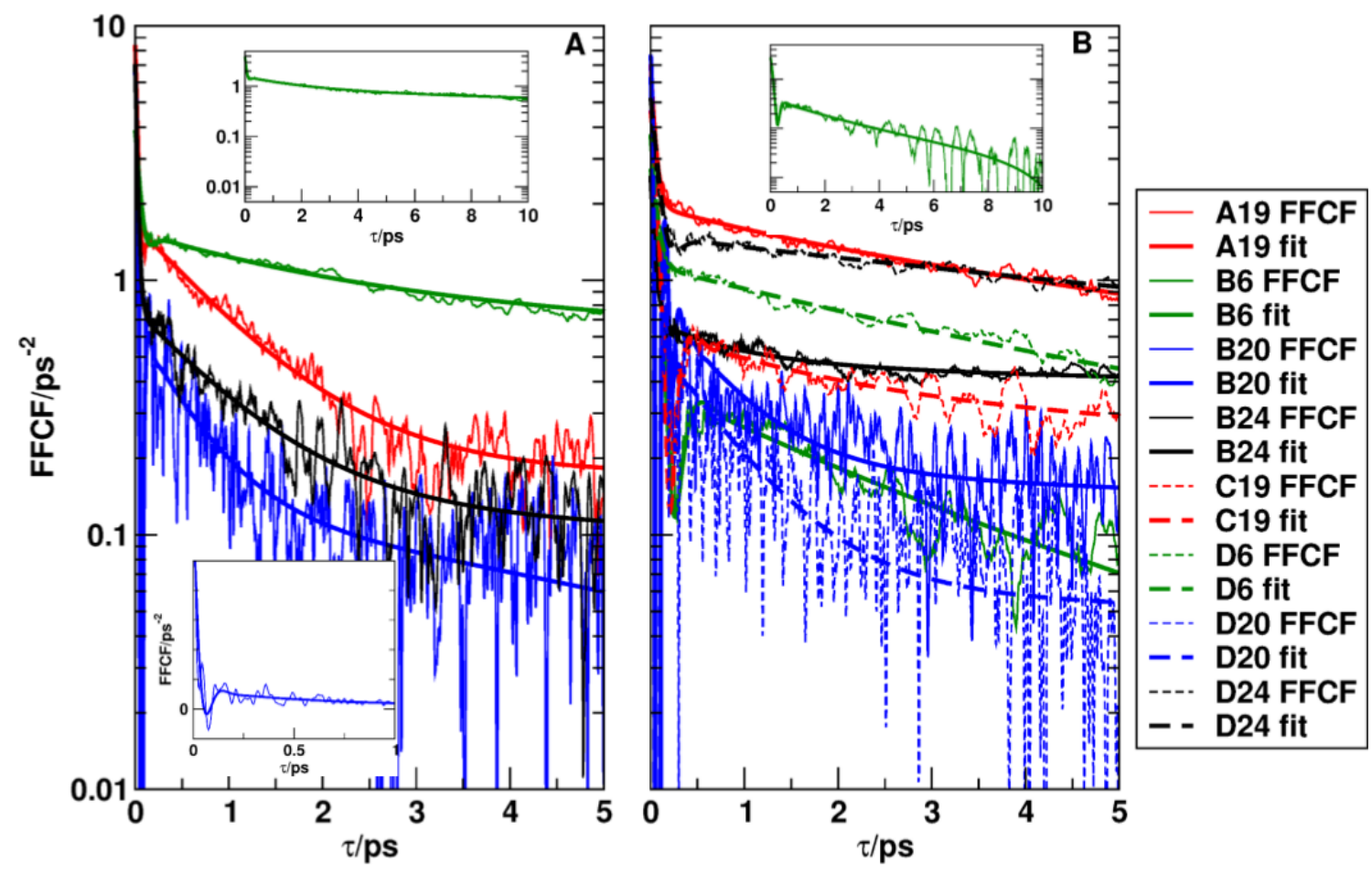

Figure 3: Frequency fluctuation correlation functions (FFCFs) for 3 selected ${ }^{12} \mathrm{C}^{16} \mathrm{O}$ probes (A19, B6 and B20) and for ${ }^{13} \mathrm{C}^{18} \mathrm{O}$ (B24) for the monomeric/dimeric forms. Solid thick lines for monomer I, dashed thick lines for monomer II (in the dimer). The raw data is shown as thin lines in the same color as the fits. Insets show the FFCF over longer times for the -CO at position B6 (blue traces) and for the first 1 ps (for B20, green trace and on linear $y$-scale) to highlight the minimum at short correlation time. See Figure 1 for location of the probes in the protein.

From the FFCFs the infrared lineshape can be directly determined by analytical integration. As for the FFCFs the 1-d infrared lineshapes differ depending on the position of the -CO label. Both, the maximum of the absorption and the full width at half maximum (FWHM) are influenced by the position of the probe along the protein chain, see Figure 4 A. In the following all infrared frequencies are reported relative to the ${ }^{12} \mathrm{C}^{16} \mathrm{O}$ frequency of NMA in solution from solving the 1-dimensional Schrödinger equation (at $1586 \mathrm{~cm}^{-1}$ ). The band centers for all ${ }^{12} \mathrm{C}^{16} \mathrm{O}$ in Figure $4 \mathrm{~A}$ are shifted to the red by around $20 \mathrm{~cm}^{-1}$. This is consistent with findings from experiments on $(\mathrm{Ala})_{3}$ in water which find the -CO stretch at 
$\sim 1600 \mathrm{~cm}^{-1}$, shifted some $25 \mathrm{~cm}^{-1}$ to the red from the absorption for NMA. ${ }^{29}$ The same observation is made when scanning along the amide-I normal mode, see Figure S2. The frequency distributions shift to the red compared to that of NMA by 10 to $20 \mathrm{~cm}^{-1}$ (for the two residues considered) but the overall distribution remains almost unchanged. The FWHMs for the lineshapes at positions B20 and B24 are comparable whereas those for B6 and A19 are larger. These are also the residues for which the FFCF decayed more slowly or even remained at a finite static value at longer correlation times.
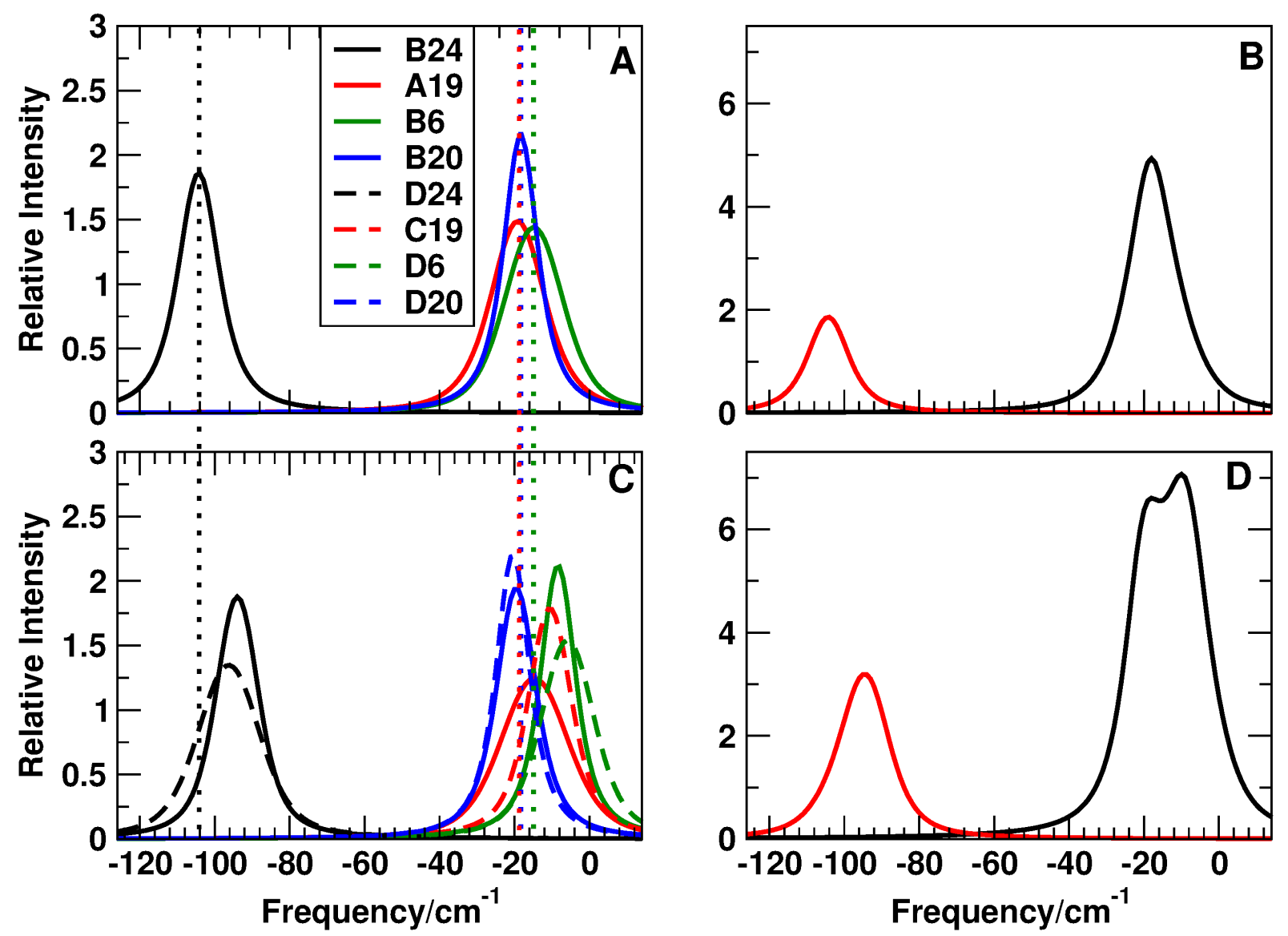

Figure 4: 1D IR absorption spectra for 3 selected ${ }^{12} \mathrm{C}^{16} \mathrm{O}$ bonds (A19, B6 and B20) and for ${ }^{13} \mathrm{C}^{18} \mathrm{O}$ (B24) for monomeric (panels A and B) and dimeric (panels C and D) insulin. See Figure 1 for locations in the protein. Solid lines for monomer I and dashed lines for monomer II. Panels $\mathrm{B}$ and $\mathrm{D}$ report the superposition of all ${ }^{12} \mathrm{C}^{16} \mathrm{O}$ lineshapes (red) compared with that of the single ${ }^{13} \mathrm{C}^{18} \mathrm{O}$ probe (black). 


\subsection{FFCF and Infrared Spectra for the Insulin Dimer}

The structural dynamics, FFCFs and 1D-infrared lineshapes were also determined for the homodimer using the same-CO probes as for the monomer. This provides information about relative shifts of the IR-absorption and changes in the FWHM. In addition, it is possible to address the question whether the dynamics of each of the monomers in the insulin dimer is symmetric, i.e. whether the crystallographic symmetry (C1) also extends to symmetry in the structural dynamics and the spectroscopic response.

Table 2: Fitting parameters of the FFCFs for $\omega(t)$ from solving the 1-d Schrödinger equation for ${ }^{12} \mathrm{C}^{16} \mathrm{O}$ and ${ }^{13} \mathrm{C}^{18} \mathrm{O}$ in monomeric and dimeric insulin for residues B24, A19, B6, B20, D24, C19, D6, D20. The data is fit to Eqs. 2 or 3 .

\begin{tabular}{l|rr|rr|rr}
\hline \hline & $\begin{array}{r}a_{1} \\
\mathrm{ps}^{-2}\end{array}$ & $\begin{array}{r}\tau_{1} \\
\mathrm{ps}\end{array}$ & $\begin{array}{r}a_{2} \\
\mathrm{ps}^{-2}\end{array}$ & $\begin{array}{r}\tau_{2} \\
\mathrm{ps}\end{array}$ & $\begin{array}{r}\Delta_{0} \\
\mathrm{ps}^{-2}\end{array}$ & $\begin{array}{r}\gamma \\
\mathrm{ps}^{-1}\end{array}$ \\
\hline B24 monomer & 6.74 & 0.02 & 0.66 & 1.01 & 0.11 & - \\
A19 monomer & 7.04 & 0.02 & 1.47 & 0.99 & 0.17 & -25.5 \\
B6 monomer & 2.40 & 0.03 & 0.92 & 2.82 & 0.59 & 0.2 \\
B20 monomer & 6.62 & 0.02 & 0.47 & 1.01 & 0.04 & 34.6 \\
\hline B24 dimer & 1.74 & 0.05 & 0.26 & 1.40 & 0.41 & - \\
A19 dimer & 4.77 & 0.04 & 1.51 & 3.96 & 0.44 & - \\
B6 dimer & 2.00 & 0.10 & 0.35 & 3.15 & 0.00 & 8.3 \\
B20 dimer & 7.52 & 0.02 & 0.38 & 1.56 & 0.13 & 38.0 \\
\hline D24 dimer & 3.54 & 0.06 & 1.41 & 11.02 & 0.05 & -0.2 \\
C19 dimer & 3.62 & 0.08 & 0.36 & 3.15 & 0.22 & -10.9 \\
D6 dimer & 2.49 & 0.06 & 0.97 & 4.09 & 0.16 & - \\
D20 dimer & 6.02 & 0.02 & 0.30 & 1.51 & 0.04 & 41.6 \\
\hline
\end{tabular}

The relevant FFCFs for monomers $\mathrm{A}$ or $\mathrm{B}$ are reported in Figure $3 \mathrm{~B}$. It is found that all FFCFs change compared to those in the isolated monomer. Typically, the time scale for the long-time decay increases (see Table 2) for all probes considered. For B6 the minimum at short times is more pronounced for the dimer compared to the monomer which is also true for B20. For A19 the decay slows down considerably in the monomer and for B24, directly located at the dimerization interface, the static component also increases considerably. 


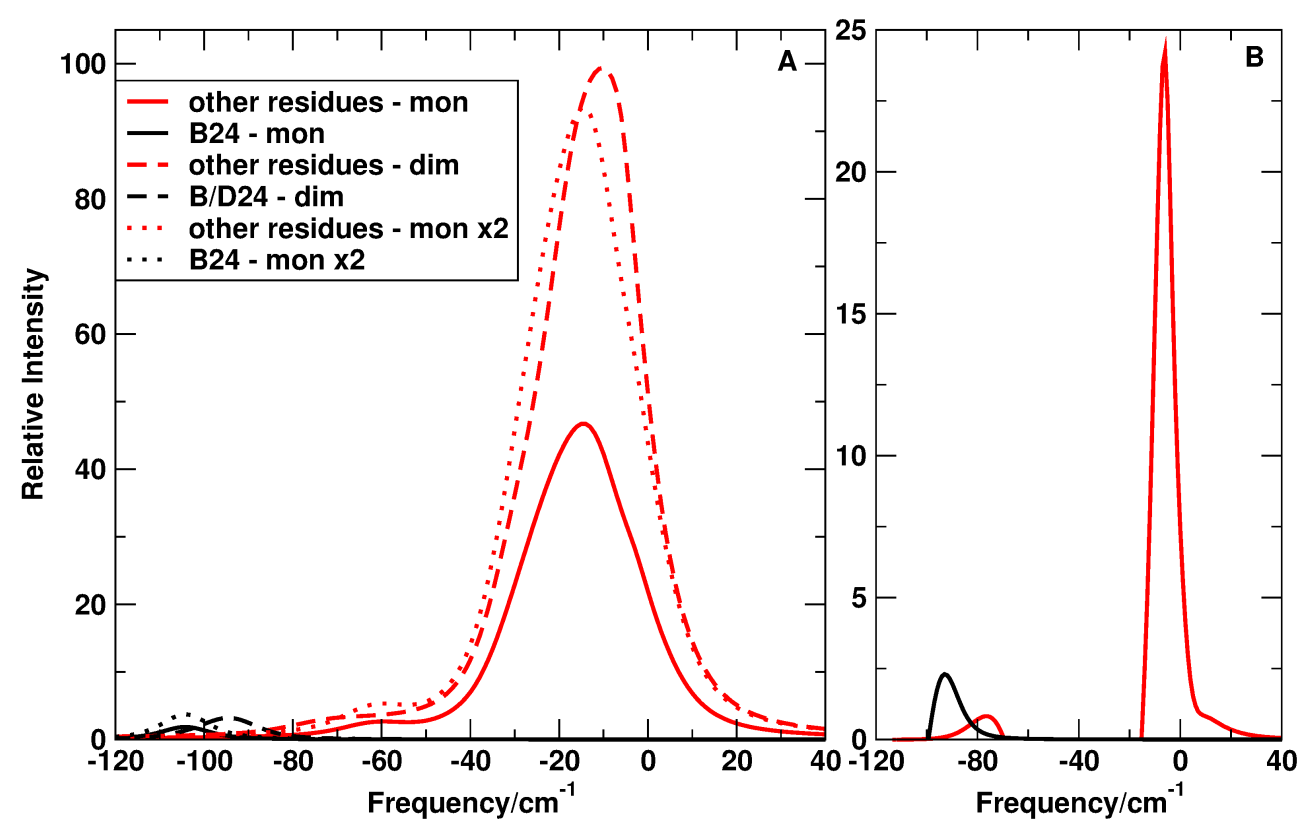

Figure 5: Panel A: The IR spectra of insulin monomer (solid line) and dimer (dashed line) from a superposition of all individual IR spectra. Also, twice the IR spectrum of the monomer is shown (dotted) which differs from the IR spectrum of the dimer and suggests an asymmetry between the two monomers in the dimer. Panel B: The difference spectrum between dimer and twice the monomer. Black traces are for B24 which is labelled.

These change in the FFCFs are also reflected in the IR spectroscopy. Both, the position of the absorption maxima and the FWHM differ in going from the monomer to the dimer. Specifically, $\left[\omega_{\max }, \mathrm{FWHM}\right]$ (both in $\mathrm{cm}^{-1}$ ) change as follows: for A19 $[-20,16] \rightarrow[[-17$, $16]$ and $[-12,16]$, for $\mathrm{B} 6[-12,19] \rightarrow[[-9,11]$ and $[-7,16]]$, for $\mathrm{B} 20[-18,12] \rightarrow[-19,12]$ and $[-20,10]]$, and for B24 $[-104,14] \rightarrow[[-95,13]$ and $[-94,23]]$, see Figures 4 A and C. The aggregate spectra for the three ${ }^{12} \mathrm{C}^{18} \mathrm{O}$ and the ${ }^{13} \mathrm{C}^{18} \mathrm{O}$ probe (at B24) for the monomer and the dimer are shown in Figures $4 \mathrm{C}$ and D. They also show that the IR-spectroscopy of the monomer and the dimer contain potentially valuable information about the aggregation state of the hormone, see also Figure 5, which establishes that the IR spectrum of the dimer differs from that of twice the monomer which should be observable experimentally.

It should be noted that the experimentally measured infrared spectrum of insulin dimer finds 
the amide-I bands at around $1640 \mathrm{~cm}^{-1}$, i.e. blue shifted relative to that found in NMA. .68 However, these experiments are carried out at low pH including DCl, $\mathrm{NaCl}$ and $20 \%$ EtOD as cosolvent. Conversely, for experiments with (Ala) $)_{3}$ in water the amide-I absorptions shift to the red by up to $20 \mathrm{~cm}^{-1} . \underline{26}$

In summary, the dynamics and infrared spectroscopy of individual -CO probes along the polypeptide changes is sensitive to the aggregation state of the hormone. In a next step, these changes are discussed in the context of the overall structural dynamics of insulin monomer and dimer.

\subsection{Structural Dynamics and Spectroscopy}

The dimerization interface of insulin involves residues B24 to B26 which are linked through hydrogen bonds to residues D26 to D24. Considering this, the change in the FFCFs and infrared spectra for the -CO probes of these residues upon dimerization are a primary target for a structural interpretation of the spectroscopy. For the monomer the -CO stretches for B25 and B26 superimpose (Figure 64) whereas that for B24 is shifted to the red because the heavy isotope was used in the simulation. Upon dimerization this changes. For the label at B24 the bands shift by 8 to $10 \mathrm{~cm}^{-1}$ to the blue and the FWHM increase somewhat, see Figure 6B. For the labels at positions B25 and B26 all maxima shift to the blue and the linewidths increase. The frequency shift ranges from 5 to $20 \mathrm{~cm}^{-1}$, consistent with findings for $(\text { Ala })_{3} \cdot{ }^{26}$ As for B24 the two symmetry-related positions in the X-ray structure split for the structural dynamics and instead of 1 band a total of 4 bands emerge. Thus, dimerization leads to noticeable changes in the IR-spectroscopy of the -CO probes depending on the changes of their environment at the interface.

Another potentially interesting region that may be affected by dimerization is around the turn involving residues B20 to B23 because these residues can interact with the end of the B- 


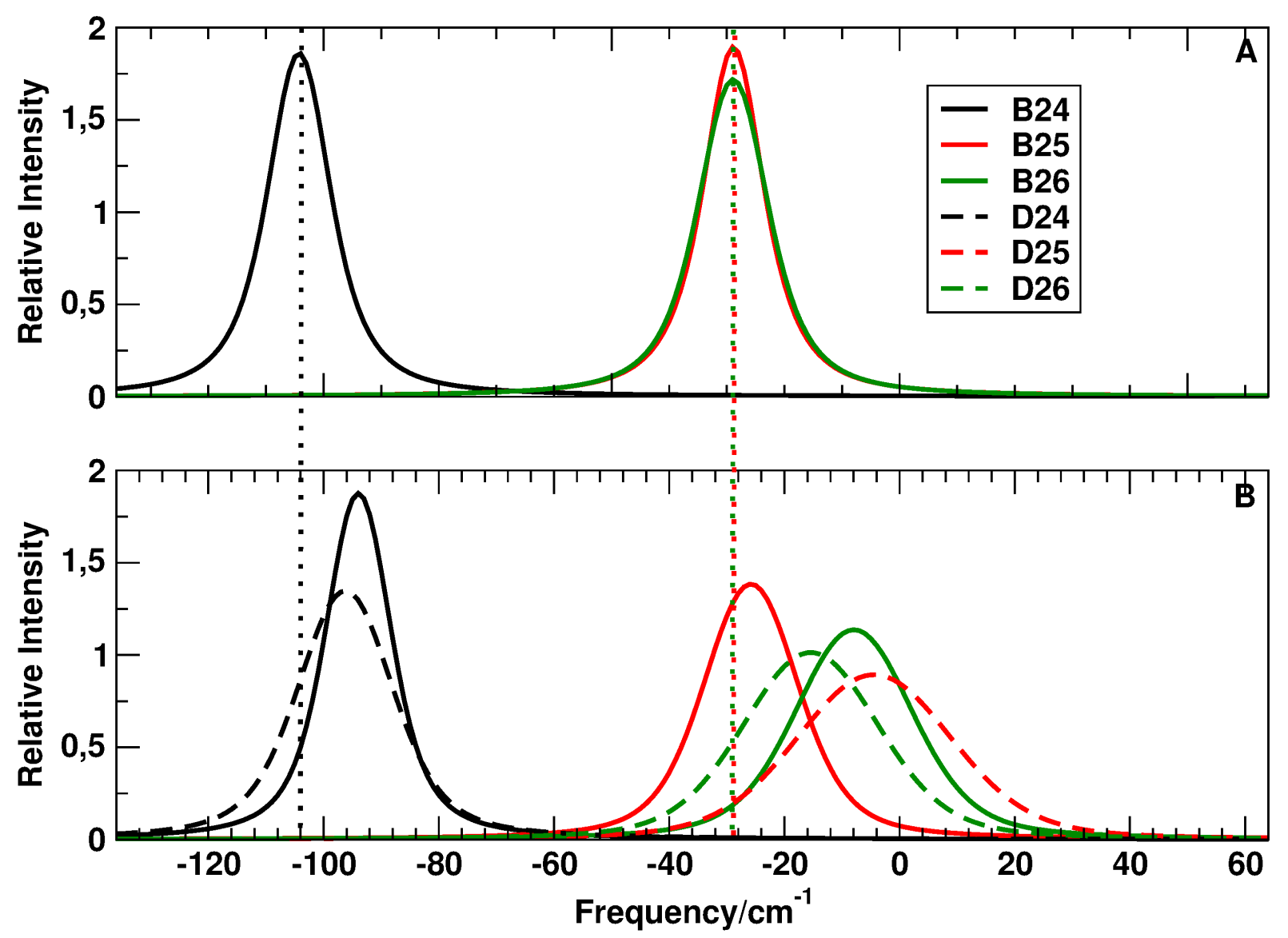

Figure 6: The IR spectra for residues B24 to B26 in the monomer (upper panel) and for the two monomers in the dimer (lower panel) for isotopically labelled B24. 
chain (residues B27 onwards), see Figure 1. These IR spectra are shown in Figure 7. Again, it is found that the spectra of the monomer and dimer do not overlap, see Figures $7 \mathrm{~A}$ and B. In addition, the IR spectra for the symmetry-related pairs in the B- and D-chains do not superimpose which suggests that the structural dynamics of the dimer is not symmetric for the two monomers in the homo-dimer. However, the effects of dimerization on the position of the frequency maxima are considerably smaller $\left( \pm 5 \mathrm{~cm}^{-1}\right)$ than those for residues B24 to B26 which are directly involved in dimerization through H-bonding for which they span \pm 25 $\mathrm{cm}^{-1}$.
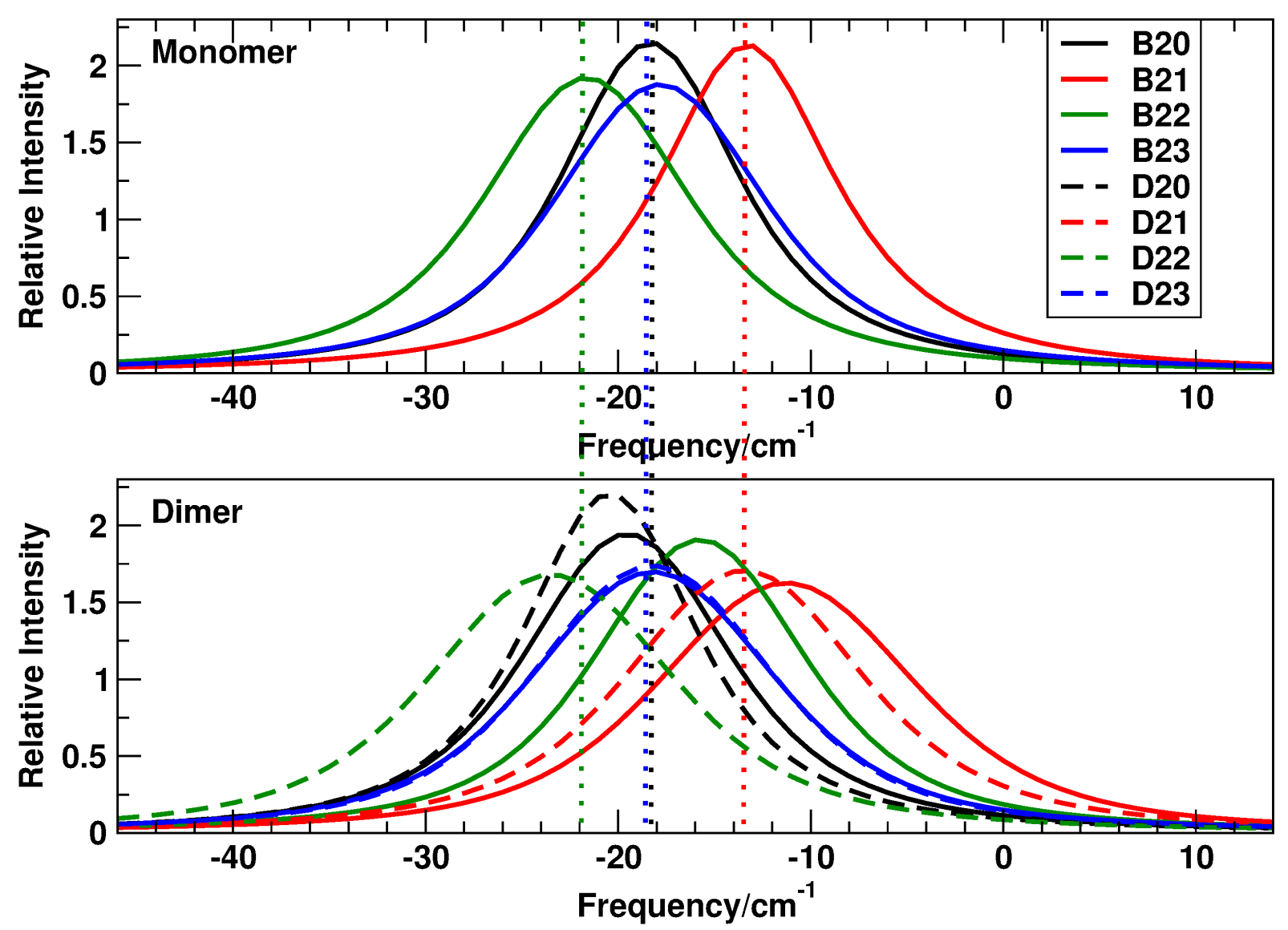

Figure 7: The IR spectra for residues B20 to B23 in the monomer (upper panel) and for the two monomers in the dimer (lower panel).

Given that the FFCFs and 1d-infrared response derived from it provide valuable informa- 
In the following typical determinants that affect the spectroscopy around a probe are considered. First, the average number of water molecules $n_{W}$ within a radius of $4 \AA$ of the label is considered depending on the position of the - $\mathrm{CO}$ along the polypeptide chain, see Figure 8. It is found that with increasing average hydration $\left\langle n_{\mathrm{W}}\right\rangle$ the infrared absorption shifts gradually to the red. This is consistent with a typical red shift of a - CO group upon interaction with the hydrogen atoms of the water molecules. However, the correlation is not particularly strong. In Figure $8 \mathrm{~A}$ a linear correlation determined from all data (red) and a line (blue) connecting the average $\left.\left(\left\langle n_{w}\right\rangle,<\omega\right\rangle\right)$ for the lowest $\left(n_{W} \sim 0\right)$ and largest $\left(n_{W} \sim 4\right)$ degree of hydration both illustrate this. Hence, the overall frequency shifts can be rationalized based on local interactions with hydration waters. The water-exposure of residues F24, F25, and Y26 changes appreciably between the monomeric and dimeric form, as expected. They are $n_{w} \geq 3$ for the monomer and reduce to $n_{w} \sim 1$ for the dimer. The fact that water can penetrate into the dimerization interface has recently been found in simulations for $\mathrm{WT}$ and mutant insulins at position B24. 13

Next, the value $C(t=0)$ of the FFCF is compared with the average number of water molecules. Again, there is a weak linear correlation (Figure 9 between the two quantities which indicates that with increasing hydration the amplitude $C(t=0)$ increases. This (weak) correlation can also be understood in a qualitative fashion because the amplitude of the FFCF is related to the interaction between the $-\mathrm{CO}$ probe and the environment which 

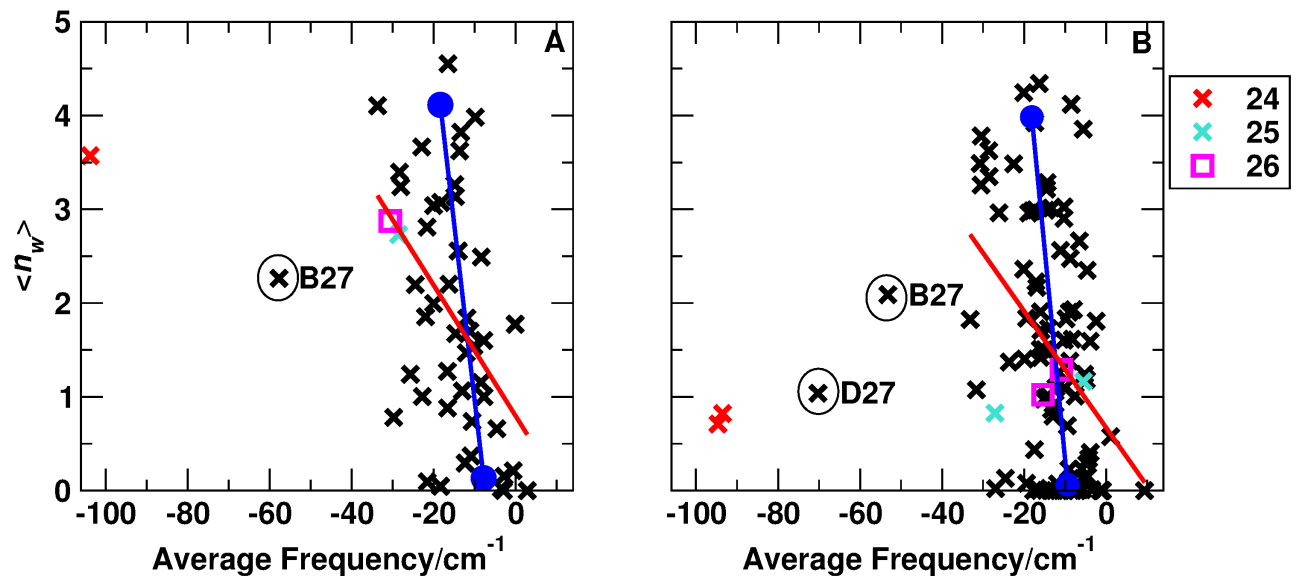

Figure 8: The maximum of the IR spectrum vs. the average number of water molecules within a $4 \AA$ radius of the $\mathrm{O}$ atom of the amide bond for the monomer. Residues B24, B25, B26 and the outliers are labelled specifically. The red line is the linear correlation for all data except for B24 and the outliers. The blue line connects the centers of gravity (blue dots) for $n_{W} \sim 0$ and $n_{w} \sim 4$. There is no ready structural explanation for the outlier at position 27.

leads to a shift of the average stretching frequency to the red, see Figure 8

Several other determinants of the spectra were compared, e.g. the maximum of the absorption band with the fluctuation of the frequency itself or with the average residence time of the water molecules (see Figure S3). However, these were not found to be related even in a qualitative fashion. In summary, there is no single, simple structural feature of the conformational dynamics that can be related in a straightforward and unambiguous manner with the frequency shift of the -CO oscillator. This is also not to be expected given the demanding environment of each of the oscillators that changes on the picosecond time scale.

A final question concerns the origin of the frequency shifts which was assessed in the following way for a typical -CO label that is surrounded by both, water and neighboring protein residues. The -CO group of the LeuB17 residue in the B16-B17-B18 (Tyr-Leu-Val) tripeptide has been chosen for this as it is surrounded by both, water and protein environments. Fifty snapshots were extracted from the MD simulations of the insulin monomer in water and pre- 
Figure 9: The value of $C(t=0)$ vs. the average number of waters within a $4 \AA$ radius of the $\mathrm{O}$ atom of the amide bond. The black and red solid lines are the linear correlations for all data except the outliers for monomer and dimer, respectively. The blue (monomer) and yellow (dimer) solid lines connecting the filled circles (center of gravity) for $n_{W} \sim 0$ and $n_{w} \sim 4$. There is no obvious explanation for the outliers (in circles and labelled).

pared in the following way. The tripeptide and all surrounding water molecules and amino acid residues within $5 \AA$ of the -CO label of LeuB17 were selected (typically 100 atoms) for each snapshot. For each snapshot the 1-dimensional potential energy surface was scanned along the -CO normal mode at the B3LYP $/ 6-31+\mathrm{G}(\mathrm{d}, \mathrm{p})$ level of theory using Gaussian09 $9^{69}$ and the transition frequency was determined by solving the 1-dimensional Schrödinger equa$\operatorname{tion}^{\sqrt[70]{0}}$ on the $a b$ initio PES. This was repeated for the systems in which (i) all water molecules, (ii) all residues surrounding the tripeptide and (iii) both, water molecules and surrounding residues were removed ("gas phase"). Thus, in total 4 frequency distributions were obtained and the frequency shifts induced by the water, amino acid residues and both were computed with respect to the gas phase frequency of the tripeptide for each snapshot. 
The results in Figure 10 show that the frequency distribution of the full system (tripeptide + water + closest protein residues, green) is shifted by $\sim-55 \mathrm{~cm}^{-1}$ to the red relative to the gas phase tripeptide frequencies. The shift induced by the closest protein residues (red) is only $\sim-10 \mathrm{~cm}^{-1}$ and is relatively narrow $\left( \pm 10 \mathrm{~cm}^{-1}\right)$ whereas that due to the water shell (blue) amounts to $\sim-50 \mathrm{~cm}^{-1}$ and is comparable in width to the green distribution. This suggests that most of the spectroscopic response for this -CO probe is due to the water shell.

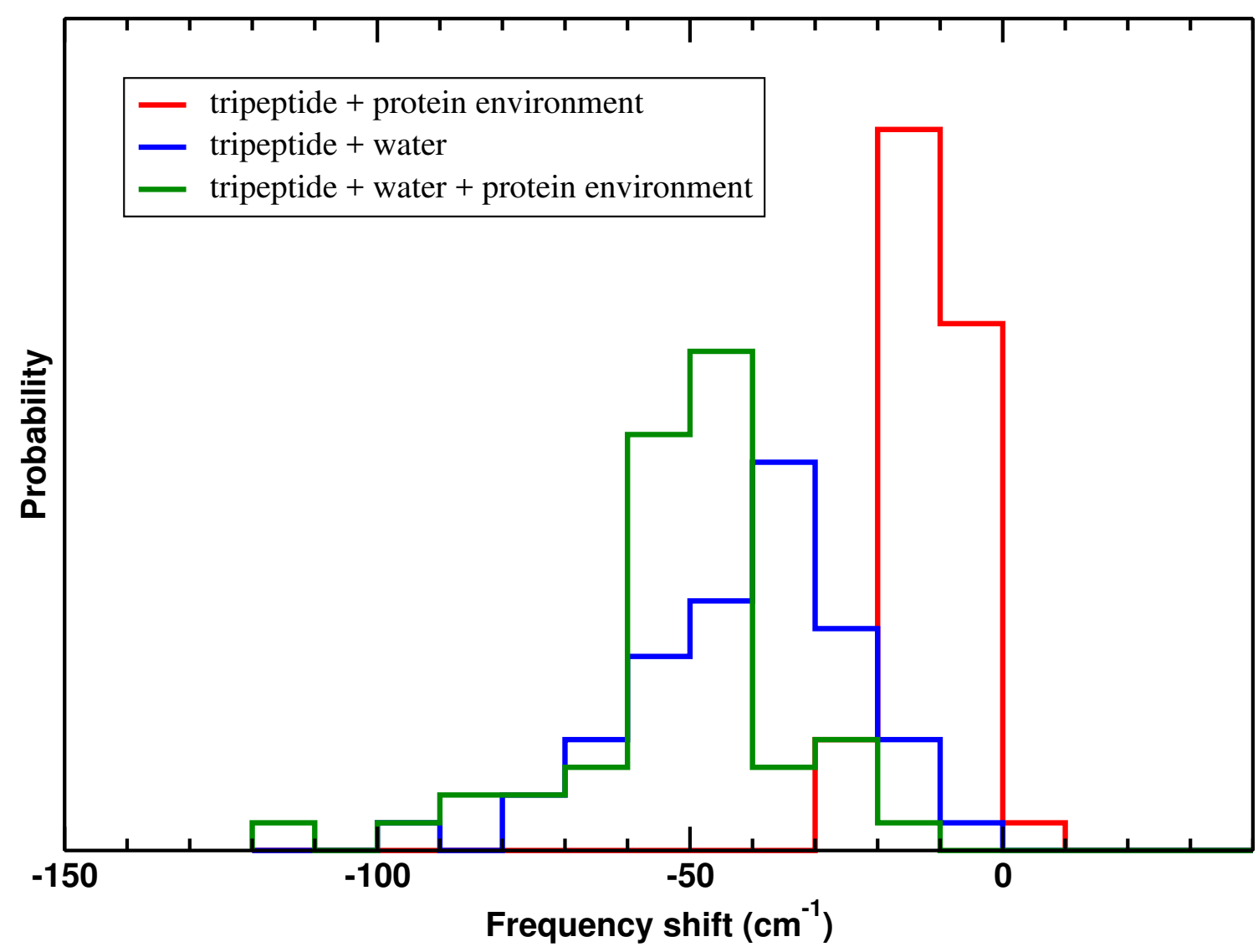

Figure 10: Distribution of frequency shifts for the -CO stretch mode of LeuB17 in B16B17-B18 obtained from 50 snapshots for the tripeptide is either surrounded by amino acid residues (red) or water molecules (blue) and both the amino acid residues and water (green). The zero of frequency is the gas phase frequency of the tripeptide for each snapshot (see text for details).

This is a quantitative assessment of the influence of the water and the protein environment 


\section{Discussion and Conclusions}

Atomistic simulations using a validated force field for the spectroscopy of -CO probes for insulin monomer and dimer establish that the -CO stretch is sensitive to both, the environmental dynamics and the aggregation state of the hormone. The -CO probe frequencies span a range of $\pm 20 \mathrm{~cm}^{-1}$ for the monomer and $\pm 25 \mathrm{~cm}^{-1}$ for the dimer, see Figure 8 . Such a magnitude is consistent with previous experiments ${ }^{71}$ and simulations. ${ }^{72}$

The dimerization of insulin has been investigated using a range of experimental techniques. Using UV-vis spectroscopy dimerization free energies ranging from $-8.2 \mathrm{kcal} / \mathrm{mol}$ to -6.6 $\mathrm{kcal} / \mathrm{mol}$ were found ${ }^{6 / 73}$ which compare with $-5.4 \mathrm{kcal} / \mathrm{mol}$ from analysis of amide-I twodimensional spectroscopy (note the different definition of the reference state in Refs. $\frac{6173}{6}$ compared to Ref. $\left.{ }^{24}\right) .24$ Thus, depending on the technique used, the results can be quite different. Furthermore, a pronounced sensitivity of the stability of the dimer to addition of cosolvent (e.g. ethanol) has been reported. 24 One of the challenges in comparing experimental and computational studies is in the solvent conditions that typically are used in spectroscopic studies where ethanol, $\mathrm{HCl}$ and $\mathrm{NaCl}$ are usually added.

It has been previously suggested ${ }^{71}$ and more recently verified ${ }^{72}$ that instead of carrying out a ligand-binding experiment it is possible to use infrared spectroscopy to characterize the binding strength of a ligand towards its target protein. Based on this finding the present work sets out to characterize the differences in the spectroscopic response of the insulin 
monomer and dimer upon aggregation in the amide-I region. This may open up an avenue to complement a thermodynamic experiment with a potentially more convenient spectroscopic characterization of the aggregation state.

With regards to dimerization it is demonstrated that the dynamics and spectroscopy of the -CO probes involved in the protein-protein contact (B24 to B26 and D24 to D26) exhibit characteristic changes. In particular, spectroscopic signatures of symmetry-related -CO probes in the X-ray structure split into two distinct bands which indicates that the structure of the insulin dimer is asymmetric when the two monomers are considered. This is also consistent with the X-ray structures which explicitly note on the asymmetry of the monomer structures within the dimer. ${ }^{174}$ The rather large number of -CO oscillators (49 for the monomer, 98 for the dimer) potentially leads to a dilution of the differential spectroscopic signatures. But it may still be sufficient to distinguish monomeric from dimeric insulin in solution. If positionally resolved information is sought, isotopically substituted -CO labels will be useful. 25

For most of the analysis, the local -CO stretch mode was sampled which led us to report frequency shifts relative to the gas phase absorption in NMA. However, it would also be possible to determine the 1-dimensional potential by scanning along the normal mode corresponding to the -CO stretch (see further above for a discussion of NMA). In order to quantify the expected changes in the absolute frequencies, this was done for $10^{5}$ snapshots for the -CO label at HisB5 and TyrB26, see Figure S2, It is found that both frequencies shift by $\sim 60 \mathrm{~cm}^{-1}$ to the blue into the region where typically absorptions of insulin in solution are found whereas the widths of the distributions, and hence the lineshapes, remain the same. Thus, in the future, it may be advantageous to determine the energy along the normal mode of the -CO stretch instead of scanning the -CO local mode.

Atomistic simulations using validated energy functions together with state-of-the art ex- 
periments provide a unique opportunity to relate the spectroscopic signatures with the un-

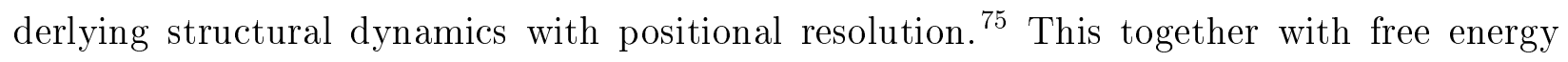
simulations ${ }^{13}$ opens up the possibility to characterize a broader range of modified insulins with respect to their thermodynamic stability of the dimers which is an essential determinant of the physiological mode-of-action of the hormone.

\section{Supporting Information Available}

Power spectra and FFCFs for NMA from vibrational mode analysis, Frequency distributions obtained from scanning along the local and normal mode of -CO for TyrB26 and HisB5, Frequency fluctuation, fluctuation of the number of water molecules and average residence time of the water molecule for insulin monomer and dimer. This material is available free of charge via the Internet at http://pubs.acs.org/.

\section{Acknowledgement}

This work was supported by the Swiss National Science Foundation grant 200021-117810, the NCCR MUST, and the University of Basel. We thank Prof. J. L. Skinner for comments on part of this manuscript and Prof. A. Tokmakoff for discussions on the solvent-sensitivity of characterizing the monomer-dimer equilibrium.

\section{References}

(1) Baker, E. N.; Blundell, T. L.; Cutfield, J. F.; Dodson, E. J.; Dodson, G. G.; Hodgkin, D. M. C.; Hubbard, R. E.; Isaacs, N. W.; Reynolds, C. D.; Sakabe, K. et al. The Structure 
of 2Zn Pig Insulin Crystals at 1.5 A Resolution. Philos. Trans. R. Soc. London, Ser. B 1988, 319, 369-456.

(2) Jørgensen, A. M. M.; Olsen, H. B.; Balschmidt, P.; Led, J. J. Solution Structure of the Superactive Monomeric Des-[Phe (B25)] Human Insulin Mutant: Elucidation of the Structural Basis for the Monomerization of Des-[Phe (B25)] Insulin and the Dimerization of Native Insulin. J. Mol. Biol. 1996, 257, 684-699.

(3) Brems, D. N.; Alter, L. A.; Beckage, M. J.; Chance, R. E.; DiMarchi, R. D.; Green, L. K.; Long, H. B.; Pekar, A. H.; Shields, J. E.; Frank, B. H. Altering the Association Properties of Insulin by Amino Acid Replacement. Protein Eng. 1992, 5, 527-533.

(4) Zoete, V.; Meuwly, M.; Karplus, M. Study of the Insulin Dimerization: Binding Free Energy Calculations and Per-Residue Free Energy Decomposition. Proteins: Struct. Funct. Bioinf. 2005, 61, 79-93.

(5) Pocker, Y.; Biswas, S. B. Self-Association of Insulin and the Role of Hydrophobic Bonding: a Thermodynamic Model of Insulin Dimerization. Biochemistry 1981, 20, 4354-4361.

(6) Strazza, S.; Hunter, R.; Walker, E.; Darnall, D. W. The Thermodynamics of Bovine and Porcine Insulin and Proinsulin Association Determined by Concentration Difference Spectroscopy. Arch. Biochem. Biophys. 1985, 238, 30-42.

(7) Hua, Q. X.; Shoelson, S. E.; Kochoyan, M.; Weiss, M. A. Receptor Binding Redefined by a Structural Switch in a Mutant Human Insulin. Nature 1991, 354, 238-241.

(8) Chen, H.; Shi, M.; Guo, Z.-Y.; Tang, Y.-H.; Qiao, Z.-S.; Liang, Z.-H.; Feng, Y.-M. Four New Monomeric Insulins Obtained by Alanine Scanning the Dimer-Forming Surface of the Insulin Molecule. Protein Eng. 2000, 13, 779-782. 
(9) DeFelippis, M. R.; Chance, R. E.; Frank, B. H. Insulin Self-Association and the Relationship To Pharmacokinetics and Pharmacodynamics. Crit. Rev. Ther. Drug Carrier Syst. 2001, 18, 201.

(10) Antolíková, E.; Žáková, L.; Turkenburg, J. P.; Watson, C. J.; Hančlová, I.; Šanda, M.; Cooper, A.; Kraus, T.; Brzozowski, A. M.; Jiráček, J. Non-Equivalent Role of Inter-And Intramolecular Hydrogen Bonds in the Insulin Dimer Interface. J. Bio. Chem. 2011, 286, 36968-36977.

(11) Jiracek, J.; Zakova, L.; Antolikova, E.; Watson, C. J.; Turkenburg, J. P.; Dodson, G. G.; Brzozowski, A. M. Implications for the Active Form of Human Insulin Based on the Structural Convergence of Highly Active Hormone Analogues. Proc. Natl. Acad. Sci. USA 2010, 10\%, 1966-1970.

(12) Zoete, V.; Meuwly, M.; Karplus, M. A Comparison of the Dynamic Behavior of Monomeric and Dimeric Insulin Shows Structural Rearrangements in the Active Monomer. J. Mol. Biol. 2004, 342, 913-929.

(13) Raghunathan, S.; El Hage, K.; Desmond, J. L.; Zhang, L.; Meuwly, M. The Role of Water in the Stability of Wild-type and Mutant Insulin Dimers. J. Phys. Chem. B 2018, 122, 7038-7048.

(14) Kristensen, C.; Kjeldsen, T.; Wiberg, F. C.; Schäffer, L.; Hach, M.; Havelund, S.; Bass, J.; Steiner, D. F.; Andersen, A. S. Alanine Scanning Mutagenesis of Insulin. J. Bio. Chem. 1997, 272, 12978-12983.

(15) Derewenda, U.; Derewenda, Z.; Dodson, E.; Dodson, G.; Bing, X.; Markussen, J. X-Ray Analysis of the Single Chain B29-A1 Peptide-Linked Insulin Molecule: A Completely Inactive Analogue. J. Mol. Biol. 1991, 220, 425-433.

(16) Vashisth, H.; Abrams, C. F. All-Atom Structural Models of Insulin Binding To the 
Insulin Receptor in the Presence of a Tandem Hormone-Binding Element. Proteins Struct., Funct. Bioinf. 2013, 81, 1017-1030.

(17) Mirmira, R.; Tager, H. Role of the Phenylalanine B24 Side Chain in Directing Insulin Interaction with Its Receptor. Importance of Main Chain Conformation. J. Bio. Chem. 1989, 264, 6349-6354.

(18) Nakagawa, S. H.; Tager, H. S. Importance of Aliphatic Side-Chain Structure at Positions 2 and 3 of the Insulin A Chain in Insulin-Receptor Interactions. Biochemistry 1992, $31,3204-3214$.

(19) Cutfield, J.; Cutfield, S.; Dodson, E.; Dodson, G.; Hodgkin, D.; Reynolds, C. Evidence Concerning Insulin Activity from the Structure of a Cross-Linked Derivative. HoppeSeyler's Z. Physiol. Chem. 1981, 362, 755-762.

(20) Ludvigsen, S.; Olsen, H. B.; Kaarsholm, N. C. A Structural Switch in a Mutant Insulin Exposes Key Residues for Receptor Binding. J. Mol. Biol. 1998, 279, 1-7.

(21) Nakagawa, S. H.; Tager, H. Role of the Phenylalanine B25 Side Chain in Directing Insulin Interaction with Its Receptor. Steric and Conformational Effects. J. Bio. Chem. 1986, 261, 7332-7341.

(22) Shoelson, S. E.; Lu, Z. X.; Parlautan, L.; Lynch, C. S.; Weiss, M. A. Mutations at the Dimer, Hexamer, and Receptor-Binding Surfaces of Insulin Independently Affect Insulin-Insulin and Insulin-Receptor Interactions. Biochemistry 1992, 31, 1757-1767.

(23) Dong, J.; Wan, Z.; Popov, M.; Carey, P. R.; Weiss, M. A. Insulin Assembly Damps Conformational Fluctuations: Raman Analysis of Amide I Linewidths in Native States and Fibrils. J. Mol. Biol. 2003, 330, 431-442.

(24) Ganim, Z.; Jones, K. C.; Tokmakoff, A. Insulin Dimer Dissociation and Unfolding 
Revealed by Amide I Two-Dimensional Infrared Spectroscopy. Phys. Chem. Chem. Phys. 2010, 12, 3579-3588.

(25) Dhayalan, B.; Fitzpatrick, A.; Mandal, K.; Whittaker, J.; Weiss, M. A.; Tokmakoff, A.; Kent, S. B. H. Efficient Total Chemical Synthesis of ${ }^{13} \mathrm{C}={ }^{18} \mathrm{O}$ Isotopomers of Human Insulin for Isotope-Edited FTIR. ChemBioChem 2016, 17, 415-420.

(26) Woutersen, S.; Hamm, P. Structure Determination of Trialanine in Water Using Polarization Sensitive Two-Dimensional Vibrational Spectroscopy. J. Phys. Chem. B 2000, $104,11316-11320$.

(27) Asplund, M.; Zanni, M.; Hochstrasser, R. Two-Dimensional Infrared Spectroscopy of Peptides by Phase-Controlled Femtosecond Vibrational Photon Echoes. Proc. Natl. Acad. Sci. USA 2000, 97, 8219-8224.

(28) Nizkorodov, S. A.; Dopfer, O.; Ruchti, T.; Meuwly, M.; Maier, J. P.; Bieske, E. J. Size Effects in Cluster Infrared Spectra: the $\nu_{1}$ Band of $\mathrm{Ar}_{n}-\mathrm{HCO}^{+}(n=1--13)$. J. Phys. Chem. 1995, 99, 17118-17129.

(29) Woutersen, S.; Pfister, R.; Hamm, P.; Mu, Y.; Kosov, D.; Stock, G. Peptide Conformational Heterogeneity Revealed from Nonlinear Vibrational Spectroscopy and MolecularDynamics Simulations. J. Chem. Phys. 2002, 117, 6833-6840.

(30) Corcelli, S.; Lawrence, C.; Skinner, J. Combined Electronic Structure/molecular Dynamics Approach for Ultrafast Infrared Spectroscopy of Dilute HOD in Liquid $\mathrm{H}_{2} \mathrm{O}$ and $\mathrm{D}_{2}$ O. J. Chem. Phys. 2004, 120, 8107-8117.

(31) Gruenbaum, S. M.; Tainter, C. J.; Shi, L.; Ni, Y.; Skinner, J. L. Robustness of Frequency, Transition Dipole, and Coupling Maps for Water Vibrational Spectroscopy. J. Chem. Theory Comput. 2013, 9, 3109-3117. 
(32) Wang, L.; Middleton, C. T.; Zanni, M. T.; Skinner, J. L. Development and Validation of Transferable Amide I Vibrational Frequency Maps for Peptides. J. Phys. Chem. B 2011, 115, 3713-3724.

(33) Hayashi, T.; Jansen, T.; Zhuang, W.; Mukamel, S. Collective Solvent Coordinates for the Infrared Spectrum of HOD in D2O Based on an Ab Initio Electrostatic Map. J. Phys. Chem. A 2005, 109, 64-82.

(34) Lee, M. W.; Carr, J. K.; Göllner, M.; Hamm, P.; Meuwly, M. 2D IR Spectra of Cyanide in Water Investigated by Molecular Dynamics Simulations. J. Chem. Phys. 2013, 139, 054506 .

(35) Cazade, P.-A.; Bereau, T.; Meuwly, M. Computational Two-Dimensional Infrared Spectroscopy without Maps: N-Methylacetamide in Water. J. Phys. Chem. B 2014, 118, $8135-8147$.

(36) Salehi, M.; Koner, D.; Meuwly, M. Vibrational Spectroscopy of $\mathrm{N}_{3}^{-}$in the Gas- and Condensed-Phase. J. Phys. Chem. B 2019, 123, 3282-3290.

(37) Ho, T.-S.; Rabitz, R. A General Method for Constructing Multidimensional Molecular Poten tial Energy Surfaces from ab Initio Calculations. J. Chem. Phys. 1996, 104, $2584-2597$.

(38) Unke, O. T.; Meuwly, M. Toolkit for the Construction of Reproducing Kernel-Based Represent ations of Data: Application to Multidimensional Potential Energy Surfaces. J. Chem. Inf. Model. 2017, 57, 1923-1931.

(39) Bloem, R.; Dijkstra, A. G.; Jansen, T. L. C.; Knoester, J. Simulation of Vibrational Energy Transfer in Two-Dimensional Infrared Spectroscopy of Amide I and Amide II Modes in Solution. J. Chem. Phys. 2008, 129. 
(40) Brooks, B. R.; Brooks III, C. L.; MackKerell Jr., A. D.; Nilsson, L.; Petrella, R. J.; Roux, B.; Won, Y.; Archontis, G.; Bartels, C.; Boresch, S. et al. CHARMM: The Biomolecular Simulation Program. J. Comput. Chem. 2009, 30, 1545-1614.

(41) J. A. MacKerell, et. al.. All-Atom Empirical Potential for Molecular Modeling and Dynamics Studies of Proteins. J. Phys. Chem. B 1998, 102, 3586-3616.

(42) Jorgensen, W. L.; Chandrasekhar, J.; Madura, J. D.; Impey, R. W. Comparison of Simple Potential Functions for Simulating Liquid Water. J. Chem. Phys. 1983, 79, $926-935$.

(43) van Gunsteren, W.; Berendsen, H. Algorithms for Macromolecular Dynamics and Constraint Dynamics. Mol. Phys. 1977, 34, 1311-1327.

(44) Swope, W. C.; Andersen, H. C.; Berens, P. H.; Wilson, K. R. A Computer Simulation Method for the Calculation of Equilibrium Constants for the Formation of Physical Clusters of Molecules: Application to Small Water Clusters. J. Chem. Phys. 1982, 76, $637-649$.

(45) Nosé, S. A Unified Formulation o the Constant Temperature Molecular-Dynamics Methods. J. Chem. Phys 1984, 81, 511-519.

(46) Hoover, W. G. Canonical Dynamics: Equilibrium Phase-Space Distributions. Phys. Rev. A 1985, 31, 1695-1697.

(47) Andersen, H. C. Molecular Dynamics Simulations at Constant Pressure and/or Temperature. J. Chem. Phys. 1980, 72, 2384-2393.

(48) Nosé, S.; Klein, M. L. Constant Pressure Molecular Dynamics for Molecular Systems. Mol. Phys. 1983, 50, 1055-1076.

(49) Hairer, E.; Lubich, C.; Wanner, G. Geometric Numerical Integration Illustrated by the Störmer/Verlet Method. Acta Numerica 2003, 12, 399-450. 
(50) Steinbach, P. J.; Brooks, B. R. New Spherical-Cutoff Methods for Long-Range Forces in Macromolecular Simulation. J. Comp. Chem. 1994, 15, 667-683.

(51) Darden, T.; York, D.; Pedersen, L. Particle Mesh Ewald: An Nlog(N) Method for Ewald Sums in Large Systems. J. Chem. Phys. 1993, 98, 10089-10092.

(52) Kramer, C.; Gedeck, P.; Meuwly, M. Atomic Multipoles: Electrostatic Potential Fit, Local Reference Axis Systems and Conformational Dependence. J. Comput. Chem. 2012, 33, 1673-1688.

(53) Bereau, T.; Kramer, C.; Meuwly, M. Leveraging Symmetries of Static Atomic Multipole Electrostatics in Molecular Dynamics Simulations. J. Chem. Theory Comput. 2013, 9, $5450-5459$.

(54) Cazade, P.-A.; Hedin, F.; Xu, Z.-H.; Meuwly, M. Vibrational Relaxation and Energy Migration of N-Methylaceamide in Water: The Role of Nonbonded Interactions. J. Phys. Chem. B 2015, 119, 3112-3122.

(55) Kubelka, J.; Keiderling, T. Ab Initio Calculation of Amide Carbonyl Stretch Vibrational Frequencies in Solution with Modified Basis Sets. 1. N-Methyl Acetamide. J. Phys. Chem. A 2001, 105, 10922-10928.

(56) Roy, R. J. Level 8.2: A Computer Program for Solving the Radial Schrödubger Equation for Bound and Quasibound Levels. University of Waterloo Chemical Physics Research Report CP-663 2014,

(57) Hamm, P.; Zanni, M. Concepts and Methods of 2D Infrared Spectroscopy; Cambridge University Press: New York, 2011.

(58) Hamm, P.; Lim, M.; Hochstrasser, R. Structure of the Amide I Band of Peptides Measured by Femtosecond Nonlinear-Infrared Spectroscopy. J. Phys. Chem. B 1998, 102, $6123-6138$. 
(59) Moller, K.; Rey, R.; Hynes, J. Hydrogen Bond Dynamics in Water and Ultrafast Infrared Spectroscopy: A Theoretical Study. J. Phys. Chem. A 2004, 108, 1275-1289.

(60) Jansen, T.; Knoester, J. A Transferable Electrostatic Map for Solvation Effects on Amide I Vibrations and Its Application To Linear and Two-Dimensional Spectroscopy. J. Chem. Phys. 2006, 124.

(61) Mayne, L. C.; Hudson, B. Resonance Raman Spectroscopy of N-methylacetamide: Overtones and Combinations of the Carbon-Nitrogen Stretch (amide II') and Effect of Solvation on the Carbon-Oxygen Double-Bond Stretch (amide I) Intensity. J. Phys. Chem. 1991, 95, 2962-2967.

(62) Kublelka, J.; Keiderling, T. A. Ab Initio Calculation of Amide Carbonyl Stretch Vibrational Frequencies in Solution with Modified Basis Sets. 1. N-Methyl Acetamide. J. Phys. Chem. A 2001, 105, 10922-10928.

(63) Song, S. S.; Asher, S. A.; Krimm, S.; Bandekar, J. Assignment of a New ConformationSensitive UV Resonance Raman Band in Peptides and Proteins. J. Am. Chem. Soc. 1988, 110, 8547-8548.

(64) Song, S. S.; Asher, S. A.; Krimm, S.; Shaw, D. K. Ultraviolet Resonance Raman Studies of Trans and Cis Peptides: Photochemical Consequences of the Twisted $\Pi^{*}$ Excited State. J. Am. Chem. Soc. 1991, 113, 1155-1163.

(65) Fang, C.; Wang, J.; Charnley, A.; Barber-Armstrong, W.; Smith, A.; Decatur, S.; Hochstrasser, R. Two-Dimensional Infrared Measurements of the Coupling Between Amide Modes of an Alpha-Helix. Chem. Phys. Lett. 2003, 382, 586-592.

(66) DeCamp, M. F.; DeFlores, L.; McCracken, J. M.; Tokmakoff, A.; Kwac, K.; Cho, M. Amide I Vibrational Dynamics of N-Methylacetamide in Polar Solvents: The Role of Electrostatic Interactions. J. Phys. Chem. B 2005, 109, 11016-11026. 
(67) Li, S.; Schmidt, J. R.; Piryatinski, A.; Lawrence, C. P.; Skinner, J. L. Vibrational Spectral Diffusion of Azide in Water. J. Phys. Chem. B 2006, 110, 18933-18938.

(68) Zhang, X.-X.; Jones, K. C.; Fitzpatrick, A.; Peng, C. S.; Feng, C.-J.; Baiz, C. R.; Tokmakoff, A. Studying Protein-Protein Binding through T-Jump Induced Dissociation: Transient 2D IR Spectroscopy of Insulin Dimer. J. Phys. Chem. B 2016, 120, $5134-5145$.

(69) Frisch, M. J.; Trucks, G. W.; Schlegel, H. B.; Scuseria, G. E.; Robb, M. A.; Cheeseman, J. R.; Scalmani, G.; Barone, V.; Mennucci, B.; Petersson, G. A. et al. Gaussian 09 Revision D.01. Gaussian Inc. Wallingford CT 2009.

(70) Colbert, D. T.; Miller, W. H. A Novel Discrete Variable Representation for Quantum Mechanical Reactive Scattering Via the Smatrix Kohn Method. J. Chem. Phys. 1992, 96, 1982-1991.

(71) Suydam, I. T.; Snow, C. D.; Pande, V. S.; Boxer, S. G. Electric Fields at the Active Site of an Enzyme : Direct Comparison of Experiment with Theory. 2006, 313, 200-204.

(72) Mondal, P.; Meuwly, M. Vibrational Stark Spectroscopy for Assessing Ligand-Binding Strengths in a Protein. Phys. Chem. Chem. Phys. 2017, 19, 16131-16143.

(73) Lord, R. S.; Gubensek, F.; Rupley, J. A. Insulin Self-Association. Spectrum Changes and Thermodynamics. Biochemistry 1973, 12, 4385-4392.

(74) Falconi, M.; Cambria, M.; Cambria, A.; Desideri, A. Structure and Stability of the Insulin Dimer Investigated by Molecular Dynamics Simulation. J. Biomol. Struct. Dyn. 2001, 18, 761-772.

(75) El Hage, K.; Brickel, S.; Hermelin, S.; Gaulier, G.; Schmidt, C.; Bonacina, L.; van Keulen, S. C.; Bhattacharyya, S.; Chergui, M.; Hamm, P. et al. Implications of Short Time Scale Dynamics on Long Time Processes. Struct. Dyn. 2017, 4, 061507. 


\section{Graphical TOC Entry}

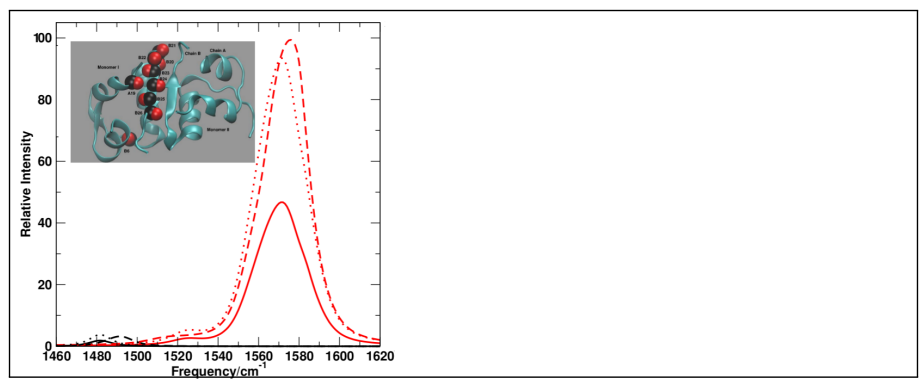

